\title{
Yangian symmetry in integrable quantum gravity
}

\author{
D. Korotkin ${ }^{1}$, H. Samtleben ${ }^{2}$ \\ Max-Planck-Institut für Gravitationsphysik, Albert-Einstein-Institut, Schlaatzweg 1, D-14473 Potsdam, \\ Germany
}

Received 2 March 1998; revised 20 April 1998; accepted 8 May 1998

\begin{abstract}
Dimensional reduction of various gravity and supergravity models leads to effectively twodimensional field theories described by gravity coupled $\mathbf{G} / \mathbf{H}$ coset space $\sigma$-models. The transition matrices of the associated linear system provide a complete set of conserved charges. Their Poisson algebra is a semi-classical Yangian double modified by a twist which is a remnant of the underlying coset structure. The classical Geroch group is generated by the Lie-Poisson action of these charges. Canonical quantization of the structure leads to a twisted Yangian double with fixed central extension at a critical level. (C) 1998 Elsevier Science B.V.
\end{abstract}

PACS: $04.60 . \mathrm{Ds} ; 04.60 \mathrm{Kz} ; 11.10 . \mathrm{Lm} ; 02.20 . \mathrm{Tw}$

Keywords: Integrable systems; Yangian symmetry; Quantum gravity; Geroch group

\section{Introduction}

The last decade has shown the important role of infinite-dimensional quantum groups in physics, providing a powerful description of the quantum symmetries of integrable models and field theories. A prominent example is the Yangian algebra $Y(\mathfrak{g})$ associated with a simple finite-dimensional Lie algebra $\mathfrak{g}$. Having turned up already in the early days of the Quantum Inverse Scattering Method [1,2] this algebra was rigorously defined within the framework of Hopf algebras by Drinfeld [3] and later on appeared to underlie many two-dimensional field theories (see Refs. [4,5] and references therein). The Yangian algebra $Y(\mathfrak{g})$ may be considered as a deformation of the positive half

\footnotetext{
' On leave of absence from Steklov Mathematical Institute, Fontanka, 27, St.Petersburg 191011, Russia.

E-mail: korotkin@aei-potsdam.mpg.de

${ }^{2}$ E-mail: henning@aei-potsdam.mpg.de 
of a loop algebra with non-trivial Hopf algebra structure. For $\mathfrak{g}=\mathfrak{g l}(N)$ it allows the representation by matrix entries $T^{a b}(w)$ of $N \times N$ matrices with exchange relations

$$
R(v-w) \stackrel{1}{T}(v) \stackrel{2}{T}(w)=\stackrel{2}{T}(w) \stackrel{1}{T}(v) R(v-w),
$$

with a rational $R$-matrix solving the Yang-Baxter equation. A deformation of the full loop algebra emerges from the Yangian double construction [6] which has been introduced in quantum field theory in $[7,8]$. Like the loop algebra this structure admits a central extension [9].

In this paper we reveal Yangian symmetries in a class of models of quantum gravity. Actually, it is the existence of this symmetry in the corresponding sectors of the classical theory which allows their complete quantization. These models descend from dimensional reduction of various gravity and supergravity theories. As effectively twodimensional theories they are given by different $\mathbf{G} / \mathbf{H}$ coset space $\sigma$-models coupled to $2 \mathrm{~d}$ gravity and a dilaton. Among them (with $\mathbf{G} / \mathbf{H}=S L(2, \mathbb{R}) / S O(2)$ ) is the two Killing vector field reduction of pure $4 \mathrm{~d}$ Einstein gravity. Its field content is given by a symmetric $2 \times 2$-matrix $g$ with unit determinant, which parametrizes the $4 \mathrm{~d}$ line element

$$
d s^{2}=e^{2 r(\rho, t)}\left(-d t^{2}+d \rho^{2}\right)+\rho g_{a b}(\rho, t) d x^{a} d x^{b} .
$$

The accompanying dilaton factor $\rho$ is a typical feature of Kaluza-Klein-like dimensional reductions; here it has already been identified with a coordinate (the so-called Weyl's canonical coordinates). Upon further truncation to diagonal $g$ this model reduces to the Einstein-Rosen waves whose quantization was studied in $[10,11]$. Other examples with higher-dimensional coset spaces $\mathbf{G} / \mathbf{H}$ come from Einstein-Maxwell systems [12,13] or maximally extended supergravity [14].

The equations of motion for all these models are given by the Ernst equation

$$
\partial_{0}\left(\rho g^{-1} \partial_{0} g\right)-\partial_{1}\left(\rho g^{-1} \partial_{1} g\right)=0,
$$

with a $\mathbf{G}$-valued matrix $g$ subject to additional restrictions which encode the $\mathbf{G} / \mathbf{H}$ coset structure. For example, for $S L(2, \mathbb{R}) / S O(2), g$ is a symmetric matrix which coincides with the matrix from (1.2) or alternatively with a matrix carrying the dualized potentials of the Ernst picture [15]. Up to the prefactor of $\rho$ the Ernst equation agrees with the dynamics of the non-linear $\sigma$-model $[16,17]$. However, it is this factor which essentially changes the properties of the model and gives rise to several new features.

In particular, it turns out that in our model the non-ultralocal contributions of the canonical Poisson brackets do not cause an obstacle for a well-defined Poisson structure between the generating functions of the integrals of motion. This allows a reformulation of the classical model in terms of a complete set of non-local conserved charges $T_{ \pm}(w)$ defined as holomorphic matrix functions in the upper and lower half of the complex plane, respectively. A consistent and essentially unique canonical quantization of the complete structure for $\mathfrak{g}=\mathfrak{s l}(N)$ leads to the following new algebra of Yangian type: 


$$
\begin{gathered}
R(v-w) T_{ \pm}^{1}(v) \stackrel{2}{T_{ \pm}}(w)=T_{ \pm}^{2}(w) T_{ \pm}^{1}(v) R(v-w) \\
R(v-w-\mathrm{i} \hbar) T_{-}^{1}(v) T_{+}^{2}(w)=T_{+}^{2}(w) T_{-}^{1}(v) R^{\eta}\left(v-w+\frac{2}{N} \mathrm{i} \hbar\right) \chi(v-w)
\end{gathered}
$$

In contrast to the known centrally extended Yangian double based on (1.1) the mixed exchange relations here contain an $R$-matrix $R^{\eta}$ which is obtained from $R(v-w) \in$ $\mathcal{U}(\mathfrak{g}) \otimes \mathcal{U}(\mathfrak{g})$ by "twisting" one of the two spaces with the algebra involution $\eta$ which characterizes the underlying coset structure. The values of the central extension as well as the rescaling factor $\chi(v)$ in (1.4) are uniquely fixed. We call this algebra a twisted centrally extended Yangian double at the critical level.

A crucial role in our model is played by the matrix product

$$
\mathcal{M}(w)=T_{+}(w) T_{-}^{\prime}(w),
$$

which in some sense behaves similar to the quantum current introduced in [9] for the normal Yangian double. This matrix coincides with the values of the original field $g$ from (1.3) at $\rho=0$. This is the symmetry axis if $\rho$ is chosen as radial coordinate; for timelike $\rho$ it describes the (cosmological) origin. Thus, the matrix $\mathcal{M}(w)$ is localized in the $2 \mathrm{~d}$ space-time though at a fixed instant of time it is defined in a highly non-local way. It provides a surprising link between the conserved charges and the physical fields. In particular, the algebra (1.4) is compatible with the symmetry of (1.5).

The present paper generalizes and gives a detailed account of the results which were partially announced in $[18,19]$. It is organized as follows. In Section 2 we give the Lagrangian formulation of the models, derive equations of motion and the canonical Poisson structure. Section 3 is devoted to analyzing the transition matrices of the associated linear system. We obtain a complete set of conserved charges and their Poisson algebra after gauge fixing of the dilaton to a spacelike and timelike coordinate, respectively. Section 4 recovers the transitive action of the Geroch group which in this framework is generated by the Lie-Poisson action of the classical algebra of charges. The heart of the paper is Section 5 where we present the quantum algebra for $\mathfrak{g}=\mathfrak{s l}(N)$. Quantizing the classical Poisson algebra we uniquely obtain (1.4) and show its consistency with all additional structures. We discuss in more detail the simplest case $\mathfrak{g}=\mathfrak{s l}(2)$ related to reduced Einstein gravity (1.2). In Section 6 we briefly summarize the solved and the remaining problems. Appendix A contains the detailed derivation of the key formula (A.11) for the Poisson bracket between transition matrices. Here we also discuss the essential difference to the principal chiral field model in flat space. Appendix B collects some useful formulas for the coordinate dependent spectral parameter.

\section{Canonical formalism}

Let $\Sigma$ be a two-dimensional Lorentzian world-sheet, parametrized by coordinates $x^{\mu}$. We introduce light-cone coordinates $x^{ \pm} \equiv x^{0} \pm x^{1}$. Let $\mathbf{G}$ be a semisimple Lie group and $\mathfrak{g}$ the corresponding Lie algebra with basis $t_{a}$. Denote by $\mathbf{H}$ the maximal compact 
subgroup of $\mathbf{G}$, characterized as the fixgroup of an involution $\eta$. Lifting $\eta$ to the algebra gives rise to the decomposition

$$
\mathfrak{g}=\mathfrak{h} \oplus \mathfrak{k} \quad \text { with } \eta(X)=\left\{\begin{array}{r}
X \text { for } X \in \mathfrak{h} \\
-X \text { for } X \in \mathfrak{k}
\end{array},\right.
$$

which is orthogonal with respect to the Cartan-Killing form.

The physical fields of the model are mappings $\mathcal{V}\left(x^{\mu}\right)$ from $\Sigma$ into the coset space $\mathbf{G} / \mathbf{H}$, i.e. they are $\mathbf{G}$-valued and exhibit the gauge freedom of right $\mathbf{H}$-multiplication. The currents $J_{\mu} \equiv J_{\mu}^{a} t_{a} \equiv \mathcal{V}^{-1} \partial_{\mu} \mathcal{V}$ allow decomposition according to (2.1):

$$
J_{\mu}=Q_{\mu}+P_{\mu}, \quad \text { with } Q_{\mu} \in \mathfrak{h}, P_{\mu} \in \mathfrak{k},
$$

such that the gauge transformations take the form

$$
Q_{\mu} \mapsto h^{-1} Q_{\mu} h+h^{-1} \partial_{\mu} h, \quad P_{\mu} \mapsto h^{-1} P_{\mu} h,
$$

with $h=h\left(x^{\mu}\right) \in \mathbf{H}$.

Let us establish the canonical setting. The dimensional reduction of gravity and supergravity theories has already and often been described in the literature (see, c.g., Refs. [14,20,21]). Hence, we here restrict ourselves to starting from the effectively twodimensional reduced theory given by $2 \mathrm{~d}$ dilaton gravity coupled to different non-linear $\mathbf{G} / \mathbf{H}$ coset space $\sigma$-models corresponding to different original theories. The remaining (reduced and gauge-fixed) Lagrangian is of the form

$$
\mathcal{L}=\frac{1}{2} \rho \operatorname{tr}\left(P_{\mu} P^{\mu}\right)=\frac{1}{2} \rho \operatorname{tr}\left(P_{0}^{2}-P_{1}^{2}\right) .
$$

In addition to the coset currents $P_{\mu}$ from (2.2) there appears a dilaton field $\rho$ related to the compactified part of the former higher-dimensional metric, which obeys the $2 \mathrm{~d}$ Laplace equation ${ }^{3}$

$$
\rho=0 \text {. }
$$

At this stage of reduction $\rho$ is no longer a canonical field, but has already been gaugefixed to a particular solution of (2.5) on the world-sheet $\Sigma$. Define its dual $\tilde{\rho}$ by ${ }^{4}$

$$
\partial_{\mu} \tilde{\rho} \equiv-\epsilon_{\mu \nu} \partial^{\nu} \rho
$$

In the sequel, we will often make use of the Weyl gauge choice, i.e. identify $\rho, \tilde{\rho}$ with the world-sheet coordinates $x^{\mu}$.

The Lagrangian (2.4) resembles the one of the principal chiral field model (PCM) $[16,17]$ with the compact group of the PCM replaced by the non-compact coset manifold $\mathbf{G} / \mathbf{H}$ and arising of the additional dilaton field $\rho$ which is in fact responsible for all

\footnotetext{
${ }^{3}$ As a field equation this equation is not derived from $(2.4)$ but from the remaining $2 \mathrm{~d}$ gravitational part of the Lagrangian which we neglect here.

${ }^{4}$ We use the convention $\epsilon_{01}=\epsilon^{10}=1$ for the antisymmetric $\epsilon$-symbol and $\eta_{\mu \nu}=\operatorname{diag}(1,-1)$ for the $2 \mathrm{~d}$ Minkowski metric.
} 
the differences that will appear in contrast to the PCM already on the classical level. Nevertheless, we may introduce the canonical framework in an analogous way. Treat the current $J_{1}(x)$ as canonical coordinates; its time derivative is expressed via the condition of vanishing curvature:

$$
\partial_{0} J_{1}=\partial_{1} J_{0}+\left[J_{1}, J_{0}\right] \equiv \nabla_{1} J_{0} .
$$

Note that the operator $\nabla_{1}$ is antisymmetric with respect to the scalar product $\left(\operatorname{tr} \int d x^{1}\right)$. The action thus reads

$$
\begin{aligned}
\frac{1}{2} \int \rho \operatorname{tr}\left(P_{\mu} P^{\mu}\right) d x^{0} d x^{1} & =\frac{1}{2} \int \rho \operatorname{tr}\left(P_{0} \nabla_{1}^{-1}\left(\partial_{0} J_{1}\right)-P_{1}^{2}\right) d x^{0} d x^{1} \\
& =-\frac{1}{2} \int \operatorname{tr}\left(\left(\partial_{0} J_{1}\right) \nabla_{1}^{-1}\left(\rho P_{0}\right)-\rho P_{1}^{2}\right) d x^{0} d x^{1} .
\end{aligned}
$$

Introduce the corresponding momenta $\pi_{J} \equiv \pi_{Q}+\pi_{P}$ with canonical Poisson structure ${ }^{5}$

$$
\left\{J_{I}^{a}(x), \pi_{J}^{b}(y)\right\}=\delta^{a b} \delta(x-y),
$$

at equal times. From the action it follows that

$$
\rho P_{0}=-\nabla_{1} \pi_{J}=-\partial_{1} \pi_{J}-\left[J_{1}, \pi_{J}\right],
$$

which according to (2.1) implies

$$
\begin{aligned}
\rho P_{0} & =-\partial_{1} \pi_{P}-\left[Q_{1}, \pi_{P}\right]-\left[P_{1}, \pi_{Q}\right], \\
0 & =-\partial_{1} \pi_{Q}-\left[Q_{1}, \pi_{Q}\right]-\left[P_{1}, \pi_{P}\right] .
\end{aligned}
$$

The first equation expresses a part of the coordinates' time derivatives in terms of the canonical momenta; the second equation defines a set of weakly vanishing (first-class) constraints

$$
\phi \equiv-\partial_{1} \pi_{Q}-\left[Q_{1}, \pi_{Q}\right]-\left[P_{1}, \pi_{P}\right] \approx 0
$$

related to the gauge transformations (2.3). They consistently close into the algebra

$$
\left\{\phi^{a}(x), \phi^{b}(y)\right\}=f^{a b} \phi^{c}(x) \delta(x-y),
$$

with structure constants $f^{a b}{ }_{c}$ from $\mathfrak{h}$.

For further calculations, we switch to the index-free tensor notation. Denote for some matrix $X^{6}$

$$
\stackrel{1}{X} \equiv X \otimes I, \quad \stackrel{2}{X} \equiv I \otimes X
$$

Define accordingly the following matrix notation of Poisson brackets [22]:

$$
\{\stackrel{1}{A}, \stackrel{2}{B}\}^{a b, c d} \equiv\left\{A^{a b}, B^{c d}\right\}
$$

\footnotetext{
${ }^{5}$ Here and in the following we denote for simplicity the spatial coordinate $x^{1}$ by $x$ only and the timelike coordinate $x^{0}$ by $t$.

${ }^{6}$ In components this takes the form $(X \otimes I)^{a b, c d} \equiv X^{a b} \delta^{c d}$ and $(I \otimes X)^{a b, c d} \equiv X^{c d} \delta^{a b}$.
} 
for matrices $A^{a b}, B^{c d}$. Let $\Omega_{\mathfrak{g}} \equiv t_{a} \otimes t^{a}$ be the Casimir element of $\mathfrak{g}$, which due to orthogonality of (2.1) allows the decomposition $\Omega_{\mathfrak{g}}=\Omega_{\mathfrak{h}}+\Omega_{\mathfrak{k}}$. The canonical brackets (2.7) in this notation become

$$
\left\{\stackrel{1}{Q}_{\mathfrak{1}}(x), \stackrel{2}{\pi}_{Q}(y)\right\}=\Omega_{\mathfrak{h}} \delta(x-y), \quad\left\{P_{P_{\mathfrak{l}}}(x), \stackrel{2}{\pi}_{P}(y)\right\}=\Omega_{\mathfrak{k}} \delta(x-y) .
$$

Eq. (2.8) now yields the Poisson brackets for the physical fields:

$$
\begin{aligned}
& \left\{P_{0}(x), \stackrel{2}{\mathcal{V}}(y)\right\}=-\frac{1}{\rho(x)} \stackrel{2}{\mathcal{V}}(x) \Omega_{\mathfrak{k}} \delta(x-y), \\
& \left\{\stackrel{1}{P_{0}}(x), \stackrel{2}{\stackrel{Q}{1}_{1}}(y)\right\}=\frac{1}{\rho(x)}\left[\Omega_{\mathfrak{k}}, \stackrel{2}{P}_{1}(x)\right] \delta(x-y), \\
& \left\{\stackrel{1}{P_{0}}(x), \stackrel{2}{P_{1}}(y)\right\}=\frac{1}{\rho(x)}\left[\Omega_{\mathfrak{k}}, \stackrel{2}{Q_{1}}(x)\right] \delta(x-y)+\frac{1}{\rho(x)} \Omega_{\mathfrak{k}} \partial_{x} \delta(x-y), \\
& \left\{\stackrel{1}{P_{0}}(x), \stackrel{2}{P_{0}}(y)\right\}=\frac{1}{\rho(x)}\left[\Omega_{\mathrm{h}}, \stackrel{2}{\phi}(x)\right] \delta(x-y) \approx 0 .
\end{aligned}
$$

The Poisson algebra of constraints $\phi(x)$ (2.10) takes the form

$$
\{\stackrel{1}{\phi}(x), \stackrel{2}{\phi}(y)\}=\left[\Omega_{\mathfrak{h}}, \stackrel{2}{\phi}(x)\right] \delta(x-y) .
$$

Via adjoint action they infinitesimally generate the gauge transformations (2.3) which obviously leave the Lagrangian (2.4) invariant.

Remark 1. The Poisson brackets (2.11) are the canonical Poisson brackets derived from the Lagrangian (2.4) which in turn descends from a consistent dimensional reduction of the higher-dimensional Lagrangian of the original theory. Thus, this Poisson structure comes from proper reduction of the symplectic structure of the original theory.

An important feature to note about the Poisson brackets (2.11) is the appearance of a non-ultralocal term in the third equation. In the known flat space integrable models the presence of such a term is a good indicator for some breakdown of the conventional techniques at later stage (see, e.g., Ref. [23] for exploring the fatal consequences of the non-ultralocal term in the PCM). However, for our model we will see that this term shows a surprisingly good behavior and in fact supports the entire further treatment.

Finally, we can discuss the dynamics of the model. The equations of motion derived from (2.4) are

$$
D^{\mu}\left(\rho P_{\mu}\right)=D_{0}\left(\rho P_{0}\right)-D_{1}\left(\rho P_{1}\right)=0,
$$

with the covariant derivative $D_{\mu} P_{\nu} \equiv \partial_{\mu} P_{\nu}+\left[Q_{\mu}, P_{\nu}\right]$. In terms of the G-valued field $g \equiv \mathcal{V} \eta(\mathcal{V})^{-1} \in \mathbf{G}$ this becomes the more familiar form of the Ernst equation [15]

$$
\partial^{\mu}\left(\rho g^{-1} \partial_{\mu} g\right)=\partial_{0}\left(\rho g^{-1} \partial_{0} g\right)-\partial_{1}\left(\rho g^{-1} \partial_{1} g\right)=0 .
$$

The Hamiltonian of the model comes out to be 


$$
H=\frac{1}{2} \int \rho \operatorname{tr}\left(P_{0}^{2}+P_{1}^{2}\right) d x .
$$

There are two points to mention about the Hamiltonian dynamics here. Note first that $H$ does not govern the possible explicit time dependence of $\rho$. This is due to the fact that in the course of the reduction leading to (2.4) the system has become non-autonomous due to the particular gauge fixing of the dilaton $\rho$ to a function with explicit time dependence. In particular, $H$ generates the equations of motion only for the fields $\left(\rho P_{0}\right)$ and $P_{1}$. Secondly, $H$ generates the dynamics of these fields only up to gauge transformations (2.3) as can be explicitly checked. This corresponds to the fact that as a Hamiltonian of a constrained system $H$ is determined only up to a linear combination of the arising first-class constraints (2.9).

\section{Poisson algebra of transition matrices}

In this and the following sections we will exploit the integrability of the model to define and explore the transition matrices to be used as the fundamental objects in the sequel. The model (2.4) is integrable in the sense that it possesses a linear system $[24,25]$, i.e. the equations of motion (2.13) appear as integrability conditions of the following linear system of differential equations:

$$
\partial_{\mu} \hat{\mathcal{V}}(x, t, \gamma)=\hat{\mathcal{V}}(x, t, \gamma) L_{\mu}(x, t, \gamma)
$$

with

$$
\begin{aligned}
L_{\mu}(x, t, \gamma) & =Q_{\mu}+\frac{1+\gamma^{2}}{1-\gamma^{2}} P_{\mu}+\frac{2 \gamma}{1-\gamma^{2}} \epsilon_{\mu \nu} P^{\nu}, \\
\gamma(x, t, w) & =\frac{1}{\rho}\left(w+\tilde{\rho}-\sqrt{(w+\tilde{\rho})^{2}-\rho^{2}}\right) .
\end{aligned}
$$

The PCM admits a similar linear system (A.3) with constant spectral parameter $\lambda$ $[26,27]$. The difference here, which is essential for the entire following treatment, is the coordinate dependence of $\gamma$ and its interplay with the underlying constant parameter $w$. Some useful and illustrative formulas are collected in Appendix B.

The existence of the linear system allows the construction of the transition matrices

$$
T(x, y, t, w) \equiv \hat{\mathcal{V}}^{-1}(x, t, \gamma(x, t, w)) \hat{\mathcal{V}}(y, t, \gamma(y, t, w)) .
$$

They satisfy

$$
\begin{aligned}
T(x, x, t, w) & =I \\
\partial_{x} T(x, y, t, w) & =-L_{1}(x, t, \gamma(x, t)) T(x, y, t, w), \\
\partial_{y} T(x, y, t, w) & =T(x, y, t, w) L_{1}(y, t, \gamma(y, t)), \\
\partial_{t} T(x, y, t, w) & =-L_{0}(x, t, \gamma(x, t)) T(x, y, t, w)+T(x, y, t, w) L_{0}(y, t, \gamma(y, t)) .
\end{aligned}
$$


Like the spectral parameter $\gamma$, the transition matrices also live on a twofold covering of the complex $w$-plane. Transition between the two sheets is performed by the involution $\eta$. Until explicitly stated, we shall in the following always consider the sheet with $\gamma \in D_{+} \cup D_{-}$inside of the unit circle (cf. Appendix B).

Note that $T(x, y, t, w)$ is uniquely determined by the first three equations of (3.2); its time dependence is a consequence of the equations of motion (2.13). If the physical currents and thus $L_{0}$ vanish sufficiently fast at spatial infinity, (3.2) already shows that the transition matrices connecting the spatial boundaries become integrals of motion. They shall in fact play the main role in the sequel.

Starting from (2.11) we can calculate the Poisson brackets between two transition matrices with pairwise distinct endpoints. This calculation differs from the related one in the PCM in two essential points. First, the underlying coset structure implies the appearance of a twist in the resulting Poisson algebra (3.10), (3.11). Second, the calculation for the PCM is known to be obstructed by certain ambiguities which arise due to the non-ultralocal contributions of the original Poisson brackets (2.11). They spoil a well-defined limit procedure to coinciding endpoints. In our model on the other hand, the coordinate dependence of the spectral parameter - caused by the coupling of the dilaton field $\rho$ - provides an intrinsic regularization of these ambiguities at the spatial boundaries. As a result, the Poisson algebra (A.11) for these transition matrices takes a tractable form that (up to the twist) is related to the well-known Yangian algebra [3]. We shall study this for fixed choices of $\rho$ and $\tilde{\rho}$ in the subsequent sections; the detailed calculation is postponed to Appendix A.

\subsection{Spacelike dilaton}

Assuming the vector field $\partial_{\mu} \rho$ to be globally spacelike, we now identify $\rho \equiv x$ with the radial coordinate $x \in[0, \infty$ [. This is a usual choice of coordinates for describing cylindrically symmetric gravitational waves $[10,11]$. The dual field $\tilde{\rho}(2.6)$ then is identified with the time $t$.

Consider the object

$$
\hat{\mathcal{V}}_{\mathrm{I}}(x, t, \gamma(w)) \equiv \mathcal{V}(x=0, t) T(0, x, t, w),
$$

with $\gamma \in D_{+} \cup D_{-}$inside of the unit circle. According to (3.2) and (B.5), this is a solution of the linear system (3.1). A closer look at the properties of the spectral parameter (cf. Appendix B) shows that it is the unique solution which is holomorphic inside of the unit circle in the $\gamma$-plane. ${ }^{7}$

Let us assume that the physical currents $J_{\mu}$ fall off sufficiently fast at spatial infinity $x \rightarrow \infty$. In this limit $\hat{\mathcal{V}}_{1}$ then becomes $t$-independent. As a function of $w$ it becomes discontinuous along the real $w$-axis (since the branch cut blows up and cuts the plane into two halves). Upon further right multiplication with $\mathcal{V}^{-1}(x=\infty, t)$ these constants

\footnotetext{
${ }^{7}$ This normalization for the solution of the linear system has e.g. been chosen in $|20|$.
} 
of motion become also invariant under the gauge transformations (2.3). We denote these charges by

$$
T_{ \pm}(w) \equiv \overline{T_{\mp}(\bar{w})} \equiv \mathcal{V}(x=0, t) T(0, \infty, t, w) \mathcal{V}^{-1}(x=\infty, t) \quad \text { for } \gamma(w) \in D_{ \pm}
$$

They will provide the new variables of the model. As functions of the constant spectral parameter $w$ the $T_{ \pm}(w)$ are holomorphic in the upper and lower half of the complex plane, respectively. We can still bring them into a more illustrative form. Starting from

$$
T_{ \pm}(w)=\mathcal{V}(x=0, t) \mathcal{P} \exp \int_{0}^{\infty} d x\left(Q_{1}+\frac{1+\gamma^{2}}{1-\gamma^{2}} P_{1}-\frac{2 \gamma}{1-\gamma^{2}} P_{0}\right) \mathcal{V}^{-1}(x=\infty, t)
$$

the $t$-independence may be exploited to calculate this expression for real $w$ at the specific value $t=-w$ (assuming regularity of the currents):

$$
T_{ \pm}(w)=\mathcal{V}(x=0, t=-w) \mathcal{P} \exp \int_{0}^{\infty} d x\left(Q_{1}(x,-w) \pm \mathrm{i} P_{0}(x,-w)\right) \mathcal{V}^{-1}(x=\infty, t)
$$

Thus, on the real $w$-axis $T_{ \pm}(w)$ naturally factorizes into the product of a real and a compact part. Let us further assume that $g \equiv \mathcal{V} \eta(\mathcal{V})^{-1}$ tends to the asymptotical value $I$ at spatial infinity $(x=\infty)$. It follows that the matrix product

$$
\mathcal{M}(w) \equiv \lim _{\epsilon \rightarrow 0}\left(T_{+}(w+i \epsilon) \eta\left(T_{-}^{-1}(w-i \epsilon)\right)\right), \quad \text { for } w \in \mathbb{R},
$$

peels off the compact part of the factorization (3.5). Thus, it coincides with the values of the original field on the symmetry axis $\rho=0$ :

$$
\mathcal{M}(w)=\mathcal{V}(x=0, t=-w) \eta\left(\mathcal{V}^{-1}(x=0, t=-w)\right)=g(x=0, t=-w) .
$$

In particular, it is real,

$$
\mathcal{M}(w)=\overline{\mathcal{M}(w)}
$$

and satisfies

$$
\mathcal{M}(w)=\eta(\mathcal{M}(w))^{-1}
$$

Vice versa, (3.6) can be interpreted as the essentially unique (Riemann-Hilbert) factorization of $\mathcal{M}$ into a product of matrices holomorphic in the upper and the lower half of the complex $w$-plane, respectively. ${ }^{8}$

\footnotetext{
${ }^{8}$ The matrix $\mathcal{M}(w)$ in fact coincides with the so-called monodromy matrix of $\hat{\mathcal{V}}_{1}$, originally introduced in |20|. It is related to the transformation behavior of $\hat{\mathcal{V}}_{1}$ between the two sheets of $\gamma$ and may in particular be extracted from $\hat{\mathcal{V}}_{1}$ already at finite $x$.
} 
Eq. (3.7) provides a physical interpretation for the new constants of motion. Having been defined as spatially non-local charges for fixed $t$, they gain a definite localization in the $2 \mathrm{~d}$ space-time at fixed $x$. Moreover, they contain the entire information about the solution: Together with the fact that $\left(\partial_{x} \mathcal{V}\right)(x=0)=0$ which follows from the equations of motion (2.13), the values on the symmetry axis $x=0$ allow us to recover the field $\mathcal{V}$ everywhere. In some sense the initial values on a spacelike surface have been transformed into initial values along a timelike surface. Thus, the $T_{ \pm}(w)$ build a complete set of constants of motion for this classical sector of solutions regular on the symmetry axis.

Their Poisson algebra is derived in Appendix A.2:

$$
\begin{aligned}
& \left\{T_{ \pm}^{1}(v), T_{ \pm}^{2}(w)\right\}=\left[\frac{\Omega_{\mathfrak{g}}}{v-w}, T_{ \pm}(v) T_{ \pm}^{2}(w)\right] \\
& \left\{T_{ \pm}^{1}(v), T_{\mp}^{2}(w)\right\}=\frac{\Omega_{\mathfrak{g}}}{v-w} T_{ \pm}(v) T_{\mp}^{2}(w)-T_{ \pm}(v) T_{\mp}^{2}(w) \frac{\Omega_{\mathfrak{g}}^{\eta}}{v-w}
\end{aligned}
$$

with $\Omega_{\mathfrak{g}}^{\eta} \equiv \Omega_{\mathfrak{h}}-\Omega_{\mathfrak{k}}$ obtained from $\Omega_{\mathfrak{g}}$ by applying $\eta$ in one of the two spaces. Eqs. (3.10) build two semi-classical copies of the Yangian algebra that is well known from other $2 \mathrm{~d}$ field theories $[4,8,5]$. By semi-classical we conventionally mean that the Poisson brackets ( 3.10 ) coincide with the commutator of the $\hbar$-graded Yangian algebra in first order in $\hbar$. The mixed relations (3.11) appear "twisted" by the involution $\eta$ with respect to those coming from the normal Yangian double. Note that whereas (3.10) remains regular at coinciding arguments, (3.11) becomes obviously singular at $v=w$. However, since $T_{+}$and $T_{-}$are defined in distinct domains, this singularity appears only in the limit on the real line and thus with a well-defined $i \epsilon$-prescription.

The matrices $\mathcal{M}(w)$ form a closed Poisson algebra:

$$
\begin{aligned}
\{\dot{\mathcal{M}}(v), \stackrel{2}{\mathcal{M}}(w)\}= & \frac{\Omega_{\mathfrak{g}}}{v-w} \mathcal{M}(v) \stackrel{2}{\mathcal{M}}(w)+\stackrel{1}{\mathcal{M}}(v) \stackrel{2}{\mathcal{M}}(w) \frac{\Omega_{\mathfrak{g}}}{v-w} \\
& -\stackrel{\prime}{\mathcal{M}}(v) \frac{\Omega_{\mathfrak{g}}^{\eta}}{v-w} \stackrel{2}{\mathcal{M}}(w)-\stackrel{2}{\mathcal{M}}(w) \frac{\Omega_{\mathfrak{g}}^{\eta}}{v-w} \stackrel{1}{\mathcal{M}}(v) .
\end{aligned}
$$

The singularity at $v=w$ is understood in the principal value sense. For consistency, it may be checked that (3.12) is indeed compatible with the symmetry (3.9) of $\mathcal{M}$.

Summarizing, the variables $\mathcal{M}(w)$ provide a new formulation of the model based on the closed Poisson algebra (3.12) and a physical interpretation according to (3.7). The further study and quantization of this structure will start from the factorization (3.6) with the corresponding Poisson algebra (3.10), (3.11).

Let us finally compute the Poisson brackets between the Hamiltonian of (2.15) with the new variables $T_{ \pm}$. Though they are integrals of motion they do not commute with $H$, since their definition makes explicit use of the time $t=\tilde{\rho}$. The standard formula

$$
\{\stackrel{1}{T}(x, y, v), \stackrel{2}{X}\}=\int d x^{\prime} \stackrel{1}{T}\left(x, x^{\prime}, v\right)\left\{L_{1}\left(x^{\prime}, \gamma\left(x^{\prime}, v\right)\right), \stackrel{2}{X}\right\} \stackrel{1}{T}\left(x^{\prime}, y, v\right),
$$


for arbitrary $X$, allows us to explicitly calculate the Poisson bracket

$$
\left\{T_{ \pm}(w), H\right\}=\partial_{w} T_{ \pm}(w) .
$$

This result also follows from a simple reasoning: due to the form (B.1) of the spectral parameter $\gamma$ and the definition (3.2), the explicit time dependence (the one not governed by $H$ ) of the $T_{ \pm}(w)$ equals their $w$-dependence. Since they are integrals of motion, their bracket with $H$ then takes the form (3.14).

\subsection{Timelike dilaton}

In this section we study the case of a globally timelike vector field $\partial_{\mu} \rho$, which allows us to identify $\rho$ with the time $t$. Accordingly, $\tilde{\rho}$ now builds the spatial coordinate $x$. The distinguished location $\rho=0$ which has played the role of the symmetry axis $x=0$ in the previous section becomes now the origin $t=0$. With periodic spatial topology, this is the setting of the so-called cosmological Gowdy models [28]. ${ }^{9}$ We will however just treat the asymptotic case $x \in]-\infty, \infty$ [. The fundamental structures of the preceding section reappear in this context from a somewhat different side. We keep the technical issues rather briefly here since they have been discussed in detail above.

Again the transition matrices $T(x, y, t, w)$ provide a solution of the linear system:

$$
\hat{\mathcal{V}}_{2}(x, t, \gamma(w)) \equiv T(-\infty, x, t, w),
$$

for $\gamma(w) \in D_{+} \cup D_{-}$inside the unit circle. It gives rise to the integrals of motion

$$
T(w) \equiv \mathcal{V}(-\infty) T(-\infty, \infty, t, w) \mathcal{V}^{-1}(\infty) .
$$

With (B.5) and (A.11) it follows that they satisfy the Poisson algebra

$$
\left\{\frac{1}{T}(v), \stackrel{2}{T}(w)\right\}=\left[\frac{\Omega_{\mathfrak{g}}}{v-w}, \frac{1}{T}(v) \stackrel{2}{T}(w)\right] .
$$

In contrast to the previous section this function is continuous in the $w$-plane (since the branch cut does not blow up in the limit $x \rightarrow \infty$ but rather moves along the real line). Moreover, they do not contain the complete information about the model. This may be seen most easily for solutions of (2.13) which are regular at the origin $t=0$. For these solutions (3.16) can be calculated in the limit $t \rightarrow 0$ where it becomes trivial. Since it is $t$-independent we arrive at

$$
T(w)=l \text {. }
$$

Thus, additional integrals of motion are required. Define

$$
\mathcal{M}(w)=\lim _{\epsilon \rightarrow 0} \hat{\mathcal{V}}_{2}(x, t, \gamma(w+\mathrm{i} \epsilon)) \eta\left(\hat{\mathcal{V}}_{2}^{-1}(x, t, \gamma(w-\mathrm{i} \epsilon))\right),
$$

which is independent of $x$ and $t$. This is the proper analog of (3.6). In fact, within the setting of the previous section definition (3.19) with $\hat{V}_{2}$ replaced by $\hat{V}_{1}$ equals $(3.6)$ : the

\footnotetext{
${ }^{9}$ See Refs. $|29,30|$ for a recent treatment of the Gowdy model in Ashtekar variables.
} 
latter one is obtained from the former one in the limit $x \rightarrow \infty$ while $\mathcal{M}$ is independent of $x$. In the case of timelike $\rho$ on the other hand, (3.19) cannot be expressed in terms of (3.16) (as is obvious from (3.18)). This is due to the fact that the limits $\epsilon \rightarrow 0$ and $x \rightarrow \infty$ do not interchange in (3.19), cf. Appendix B.

For the solutions regular at the origin $t=0, \mathcal{M}(w)$ can again be calculated more explicitly. Since $\mathcal{M}(w)$ is independent of $x$ and $t$, we may evaluate it at the branch point $x \equiv t-w$ and subsequently perform the limit $t \rightarrow 0$. It is important to keep $x=t-w$ during the limit process, since otherwise $\mathcal{M}(w)$ does not behave smoothly. This yields

$$
\begin{aligned}
\mathcal{M}(w) & =\lim _{t \rightarrow 0, x=w^{\prime}-t}\left[\mathcal{P} \exp \int_{-\infty}^{x} d x L_{1}(x, \gamma) \mathcal{P} \exp \int_{-\infty}^{x} d x \eta\left(-L_{1}(x, \gamma)\right)\right] \\
& =\mathcal{V}(x=-w, t=0) \eta\left(\mathcal{V}^{-1}(x=-w, t=0)\right)
\end{aligned}
$$

Thus, $\mathcal{M}(w)$ again coincides with the values of the physical field $g$ at $\rho=0$.

The set of $\mathcal{M}(w)$ together with their canonical Poisson structure (3.12) provides a proper set of fundamental variables to parametrize the phase space of solutions of (2.13) regular at the origin $t=0$. Note that the canonical formulation obviously fails to cope with describing this truncated phase space: At $t=0$ this framework breaks down with the vanishing Lagrangian (2.4), whereas at finite $t$ the condition of regularity at $t=0$ poses highly non-trivial implicit relations between the canonical coordinates and the momenta.

After some calculation, the general form of the Poisson brackets (A.11) further yields the same Poisson algebra (3.12) as in the previous section for the matrices $\mathcal{M}(w)$. Via the Riemann-Hilbert decomposition of $\mathcal{M}$ discussed above one may then further implicitly obtain the matrices $T_{ \pm}$with Poisson structure (3.10), (3.11). Thus, together with (3.20) for solutions regular at $\rho=0$ the final situation appears rather similar to the previous section. This further stresses the fundamental meaning of the Poisson structure (3.12).

Let us finally look at the results of this section from another very intriguing point of view. In the setting with a spacelike dilaton addressed in the previous section we could have derived a Poisson structure not with respect to the time $t$ but with respect to the radius $x .{ }^{10}$ The calculations of this section show that these two Poisson structures of the same model coincide for the values of the original fields on the symmetry axis $x=0$. Since these initial values provide a complete set of observables the symplectic structures are essentially equivalent. It is then tempting to speculate about further exploiting the fundamental structure (3.12) even in the case of a timelike dimensional reduction,

I" In a covariant theory this is a rather natural idea which has been discussed in particular to describe static settings [31]. For the Schwarzschild black hole, e.g., one might throw doubt upon the distinct role of time in the canonical formalism since $x$ and $t$ change their character being space- and timelike inside of the horizon, respectively. 
i.e. the reduction to stationary axisymmetric space-times, where the canonical time is no longer present.

\section{The Geroch group}

With the integrals of motion $T_{ \pm}(w)$ identified in the previous section, one can now study the symmetries they generate via their adjoint action in the canonical Poisson structure. This yields an explicit realization [18] of the Geroch group [32] with the underlying Yangian algebra $(3.10),(3.11)$. The transformations which close into an affine algebra (the loop algebra $\hat{\mathfrak{g}}$ ) do not preserve the symplectic structure. This is a particular example of the Lie-Poisson action of dressing groups generated by the transition matrices of integrable models [33-35]. For the integrable models studied so far within the framework of the quantum inverse scattering method the integrals of motion are generated by the eigenvalues of the transition matrices. Here in contrast the transition matrices $T_{ \pm}(w)$ themselves are conserved charges.

Let $\Lambda(w) \in \mathfrak{g}$ be an algebra-valued function which is regular along the real $w$-axis and vanishes at $w \mapsto \infty$. Choose a path $\ell=\ell_{+} \cup \ell_{-}$encircling the real $w$-axis, such that $\ell_{ \pm} \in H_{ \pm}$and $A(w)$ is holomorphic inside the enclosed area. Define

$$
S[\Lambda] \equiv \operatorname{tr}\left(\int_{\ell_{+}} \frac{d v}{2 \pi \mathrm{i}} T_{+}^{-1}(v) A(v) \operatorname{ad}_{T_{+}\left({ }^{\prime}\right)}+\int_{\ell_{-}} \frac{d v}{2 \pi \mathrm{i}} T_{-}^{-1}(v) \Lambda(v) \operatorname{ad}_{T_{-}\left({ }^{\prime}\right)}\right),
$$

where "ad" denotes the adjoint action via the canonical Poisson structure. Since (3.10), (3.11) yield

$$
\left\{T_{ \pm}^{1}(v), \stackrel{2}{\mathcal{M}}(w)\right\}=\left(\Omega_{\mathfrak{g}} \stackrel{2}{\mathcal{M}}(w)-\stackrel{2}{\mathcal{M}}(w) \Omega_{\mathfrak{g}}^{\eta}\right) \frac{\stackrel{1}{T_{ \pm}}(v)}{v-w}
$$

we obtain the symmetry action

$$
S[\Lambda] \mathcal{M}(w)=\Lambda(w) \mathcal{M}(w)-\mathcal{M}(w) \eta(\Lambda(w)) .
$$

This is the known infinitesimal action of the Geroch group on the matrix $\mathcal{M}$ [20]. Even though the symmetry action on the fields is highly non-linear (cf. (4.4) and (4.5) below), on the axis $\rho=0$ it linearizes to (4.2) and allows explicit "exponentiation" to finite transformations. This in particular shows transitivity of the Geroch group in the sector of solutions regular on the axis $\rho=0$.

The transformations (4.2) form the loop algebra $\hat{\mathrm{g}}$, as follows also directly after some calculation from (4.1), (3.10) and (3.11):

$$
\left[S\left[\Lambda_{1}\right], S\left[\Lambda_{2}\right]\right]=S\left[\left[\Lambda_{1}, \Lambda_{2}\right]\right] \text {. }
$$

The explicit action of $S[\Lambda]$ on the physical fields $\mathcal{V}$ follows from (3.13), 


$$
\begin{aligned}
S[\Lambda] \mathcal{V}(x) & =\int_{\ell} \frac{d v}{2 \pi \mathrm{i}}\left(\frac{2 \gamma}{\rho\left(1-\gamma^{2}\right)} \mathcal{V}(x)\left[\hat{\mathcal{V}}^{-1}(x, \gamma(v)) \Lambda(v) \hat{\mathcal{V}}(x, \gamma(v))\right]_{\mathfrak{k}}\right) \\
& =-\int_{\gamma(\ell)} \frac{\gamma d \gamma}{2 \pi \mathrm{i}}\left(\mathcal{V}(x)\left[\hat{\mathcal{V}}^{-1}(x, \gamma(v)) \Lambda(v) \hat{\mathcal{V}}(x, \gamma(v))\right]_{\mathfrak{k}}\right),
\end{aligned}
$$

with the algebra projection $[\cdot]_{k}$ corresponding to the decomposition (2.1) and where $\hat{\mathcal{V}}$ should be replaced by $\hat{\mathcal{V}}_{1}$ or $\hat{\mathcal{V}}_{2}$ respectively, depending on the choice of the dilaton $\rho$. The corresponding transformation of the currents $P_{ \pm} \equiv \frac{1}{2}\left(P_{0} \pm P_{1}\right)$ reads

$$
S[\Lambda] P_{ \pm}(x)=\int_{\ell} \frac{d v}{2 \pi \mathrm{i}}\left(\left[\frac{2 \gamma}{\rho(1 \pm \gamma)^{2}}\left[\hat{\mathcal{V}}^{-1} \Lambda \hat{\mathcal{V}}\right]_{h}, P_{ \pm}(x)\right] \mp \frac{4 \gamma^{2} \partial_{ \pm} \rho\left[\hat{\mathcal{V}}^{-1} \Lambda \hat{\mathcal{V}}\right]_{\mathrm{\ell}}}{\rho^{2}(1 \pm \gamma)^{2}\left(1-\gamma^{2}\right)}\right)
$$

Equivalent forms of the infinitesimal symmetry transformations of the Geroch group have been stated in [36-38,21]. For example, it is easy to check that the single symmetry transformations as they are made explicit in [21] may be obtained from the closed form (4.5) by means of a Taylor expansion around $w=\infty$.

We can go further and calculate the action of $S[A]$ on the solution of the linear system $\hat{\mathcal{V}}$. Evaluating the key formula (A.11) according to the definitions (4.1) and (3.3) leads to

$$
S[\Lambda] \hat{\mathcal{V}}(x, \gamma(w))=\Lambda(w) \hat{\mathcal{V}}(x, \gamma(w))-\hat{\mathcal{V}}(x, \gamma(w)) \Upsilon(\Lambda, x, \gamma(w)),
$$

with

$$
\begin{aligned}
r(\Lambda, x, \gamma(w))= & \int_{\ell} \frac{d v}{2 \pi \mathrm{i}(v-w)}\left[\hat{\mathcal{V}}^{-1} A \hat{\mathcal{V}}\right]_{\mathfrak{h}} \\
& +\frac{1-\gamma^{2}(w)}{\gamma(w)} \int_{\ell} \frac{d v}{2 \pi \mathrm{i}(v-w)} \frac{\gamma(v)}{1-\gamma^{2}(v)}\left[\hat{\mathcal{V}}^{-1} A \hat{\mathcal{V}}\right]_{\mathfrak{\ell}} .
\end{aligned}
$$

The path $\ell$ of the remaining integrals encircles the branch cut on the real $w$-axis (cf. Appendix B).

The formula (4.6) shows an interesting pattern which has been used for the traditional realization of the Geroch group via the linear system [14,20,21]. The matrix $A(w)$ depends on the constant spectral parameter $w$ and parametrizes the symmetry algebra $\hat{\mathfrak{g}}$. In contrast, $r(\Lambda, x, \gamma)$ depends on the variable spectral parameter $\gamma$ and satisfies

$$
\Upsilon(x, \gamma)=\eta\left(r\left(x, \gamma^{-1}\right)\right) \equiv \eta^{\infty}(\Upsilon(x, \gamma))
$$

i.e. it is invariant under the generalized involution $\eta^{\infty}$ which defines the so-called algebra $\mathfrak{h}^{\infty}$ as a subalgebra of $\hat{\mathfrak{g}}$ with affine parameter $\gamma$ (see Ref. [39] for a precise discussion). Recall that the linear system (3.1) determines the solution $\hat{\mathcal{V}}$ up to left multiplication with a $w$-dependent matrix. This freedom has been fixed by choosing the 
solution (3.3) holomorphic inside of the unit circle in the $\gamma$-plane. The left multiplication with an arbitrary $\Lambda(w)$ in (4.6) violates this so-called "generalized triangular gauge" (unless $\Lambda(w)$ is constant). It requires the compensating right multiplication with a unique $r(A, x, \gamma)$ from $\mathfrak{h}^{\infty}$ to restore the holomorphy inside of the unit circle. This has been the traditional non-linear realization of the Geroch group; formula (4.7) indeed provides a closed expression for the compensating $\mathfrak{h}^{\infty}$ rotation.

From (3.14) we finally directly obtain the action of $S[A]$ on the Hamiltonian $H$ :

$$
S[\Lambda] H=\operatorname{tr}\left(\int_{\ell_{+}} \frac{d v}{2 \pi \mathrm{i}} \Lambda(v) \partial_{t} T_{+} T_{+}^{-1}(v)+\int_{\ell_{-}} \frac{d v}{2 \pi \mathrm{i}} \Lambda(v) \partial_{v} T_{-} T_{-}^{-1}(v)\right),
$$

in accordance with the formula derived in [21].

Definition (4.1) illustrates that the action of the Geroch group is not symplectic but Lie-Poisson, i.e. it does not preserve the Poisson structure on the phase space but on the direct product of the phase space with the symmetry group. Its role in the quantum theory remains to be elaborated, see Ref. [35] for a general discussion. In our model we could alternatively consider the pure symplectic action of the generators $T_{ \pm}(w)$ via Poisson bracket since they are integrals of motion themselves. However, though they certainly act symplectic this action allows neither explicit exponentiation nor a closed form of the commutator algebra in contrast to (4.2) and (4.3).

\section{Quantization: A twisted Yangian double}

So far, we have achieved a complete reformulation of the classical model (2.4) in terms of the transition matrices as new fundamental variables providing a complete set of integrals of motion. This formulation reveals integrability and the classical symmetries in a beautiful way. We can now proceed with canonical quantization of the Poisson algebra derived in the previous sections.

\section{5.l. $\mathfrak{g}=\mathfrak{s l}(N)$}

For the sequel we specify the algebra $\mathfrak{g}$ to be $\mathfrak{s l}(N)$, i.e. $\eta(X)=-X^{t}$ and $\mathfrak{h}=\mathfrak{s o}(N)$. Let us recall the classical algebra of integrals of motion (3.10), (3.11). For $\mathfrak{g}=\mathfrak{s l}(N)$ it is $\Omega_{\mathfrak{s} \mid(N)}=\Pi_{N}-\frac{1}{N} I$ with the $N^{2} \times N^{2}$ permutation operator $\Pi_{N}$ :

$$
\left(\Pi_{N}\right)^{a b, c d}=\delta^{a d} \delta^{b c} .
$$

Accordingly we define its twisted analog $\Pi_{N}^{\eta}$ by ${ }^{11}$

$$
\left(\Pi_{N}^{\eta}\right)^{a b, c d} \equiv\left(-\Pi_{N}^{\tilde{f}}+\frac{2}{N} I\right)^{a b, c d} \equiv-\delta^{a c} \delta^{b d}+\frac{2}{N} \delta^{a b} \delta^{c d}
$$

\footnotetext{
"The transposition $I_{N}^{\tilde{r}}$ here amounts to transposing just one of the two spaces in which $\Pi_{N}$ lives.
} 
The Poisson algebra (3.10), (3.11) then takes the form

$$
\begin{aligned}
& \left\{T_{ \pm}^{1}(v), T_{ \pm}^{2}(w)\right\}=\left[\frac{\Pi_{N}}{v-w}, T_{ \pm}(v) T_{ \pm}^{2}(w)\right] \\
& \left\{T_{ \pm}^{1}(v), T_{\mp}^{2}(w)\right\}=\frac{\Pi_{N}}{v-w} T_{ \pm}^{1}(v) T_{\mp}^{2}(w)-T_{ \pm}(v) T_{\mp}^{2}(w) \frac{\Pi_{N}^{\eta}}{v-w},
\end{aligned}
$$

The $T_{ \pm}(w)$ are related by complex conjugation (3.4) and further restricted by the condition $T_{ \pm}(w) \in \mathbf{G}$ :

$$
\operatorname{det} T_{ \pm}(w)=1
$$

and the additional property (3.9)

$$
\mathcal{M}(w)=T_{+}(w) T_{-}^{t}(w)=T_{-}(w) T_{+}^{t}(w)=\mathcal{M}^{t}(w) .
$$

Quantization of the model in terms of these variables now amounts to replacing (5.1), (5.2) by corresponding commutator relations of an $\hbar$-graded algebra, such that these relations are compatible with the quantum analogs of (5.3) and (5.4). It admits the following essentially unique solution: ${ }^{12}$

The quantization of the presented model for $\mathfrak{g}=\mathfrak{s l}(N)$ is given by the $*$-algebra generated by the matrix entries of $N \times N$ matrices $T_{ \pm}(w)$ subject to the exchange relations

$$
\begin{aligned}
R(v-w) & T_{ \pm}^{1}(v) T_{ \pm}^{2}(w)=T_{ \pm}^{2}(w) T_{ \pm}^{1}(v) R(v-w) \\
R(v-w-\mathrm{i} \hbar) & T_{-}^{1}(v) T_{+}^{2}(w)=T_{+}^{2}(w) T_{-}^{1}(v) R^{\eta}\left(v-w+\frac{2}{N} \mathrm{i} \hbar\right) \chi(v-w),(5.6
\end{aligned}
$$

with

$$
R(v) \equiv v I-\mathrm{i} \hbar I_{N}, \quad R^{\eta}(v) \equiv v I-\mathrm{i} \hbar \Pi_{N}^{\eta}, \quad \chi(v) \equiv \frac{\Gamma\left(\frac{-\mathrm{i} \hbar-l^{\prime}}{N \mathrm{i} \hbar}\right) \Gamma\left(\frac{(N+2) \mathrm{i} \hbar-v^{\prime}}{N \mathrm{i} \hbar}\right)}{\Gamma\left(\frac{-l^{\prime}}{N \mathrm{i} \hbar}\right) \Gamma\left(\frac{(N+1) \mathrm{i} \hbar-l^{\prime}}{N \mathrm{i} \hbar}\right)} .
$$

The condition of unit determinant (5.3) is replaced by the quantum determinant

$$
\begin{aligned}
\operatorname{qdet} T_{ \pm}(w) \equiv & \sum_{\sigma \in \mathfrak{S}_{N}} \operatorname{sgn}(\sigma) T_{ \pm}^{1 \sigma(1)}(w-(N-1) \mathrm{i} \hbar) \\
& \times T_{ \pm}^{2 \sigma(2)}(w-(N-2) \mathrm{i} \hbar) \ldots T_{ \pm}^{N \sigma(N)}(w) \\
= & 1,
\end{aligned}
$$

and the quantum form of the symmetry (5.4) is given by

\footnotetext{
${ }^{12}$ For simplicity we use the same notation for the classical fields and the quantum operators. This should not lead to any confusion.
} 


$$
\mathcal{M}(w) \equiv T_{+}(w) T_{-}^{t}(w)=\left(T_{+}(w) T_{-}^{t}(w)\right)^{t},
$$

where transposition here simply refers to the $N \times N$ matrix entries. The $*$-operation is defined by

$$
T_{+}(w)^{*} \equiv T_{-}(\bar{w}),
$$

and builds a conjugate-linear anti-multiplicative automorphism of the algebra (5.5)(5.9).

Denote by $Y_{ \pm}$the algebra generated by the $T_{ \pm}(w)$, respectively, with exchange relations (5.5). These are two copies of the well-known Yangian algebra [3] which provide the unique quantization of the Poisson algebras given by (5.1). Compatibility with associativity is equivalent to the Yang-Baxter equation for $R$ :

$$
R_{12}(u-v) R_{13}(u-w) R_{23}(v-w)=R_{23}(v-w) R_{13}(u-w) R_{12}(u-v) .
$$

The corresponding compatibility of the mixed relations $(5.6)$ with associativity is equivalent to a modified (twisted) Yang-Baxter equation for $R^{\eta}$ :

$$
R_{12}^{\eta}(u-v) R_{13}^{\eta}(u-w) R_{23}(v-w)=R_{23}(v-w) R_{13}^{\eta}(u-w) R_{12}^{\eta}(u-v) .
$$

Validity of this equation follows from the relation

$$
R^{\eta}\left(v+\frac{2}{N} \mathrm{i} \hbar\right)=-(R(-v))^{\tilde{t}}
$$

and (5.11) by applying transposition in the first space. Moreover, (5.12) remains valid under a shift of the argument in $R^{\eta}(v)$ as well as under rescaling it with a factor $\chi$. Hence, whereas the exchange relations for $Y_{ \pm}$are uniquely given by (5.5) [3], the most general ansatz for the mixed exchange relations (5.6) is

$$
R\left(v-w+c_{1} \mathrm{i} \hbar\right) T_{-}^{1}(v) T_{+}^{2}(w)=T_{+}^{2}(w) T_{-}^{1}(v) R^{\eta}\left(v-w+c_{2} \mathrm{i} \hbar\right) \chi(v-w) .
$$

The resulting algebra must respect the symmetry (5.9) of $\mathcal{M}(w)$. More precisely we demand that

$$
\left(Y_{+} \oplus Y_{-}\right) \mathcal{I}=\mathcal{I}\left(Y_{+} \oplus Y_{-}\right),
$$

where $\mathcal{I} \subset \mathcal{U}\left(Y_{+} \oplus Y_{-}\right)$is spanned by the antisymmetric matrix entries of $\mathcal{M}$. Relation (5.15) ensures that the symmetry of $\mathcal{M}$ may be consistently imposed without implying further relations. Eq. (5.15) is not influenced by the choice of $\chi$ but uniquely determines the values of the $c_{j}$ in $(5.14)$ to be

$$
c_{1}=-1, \quad c_{2}=\frac{2}{N} .
$$

At these values the exchange relations between $T_{ \pm}$and $\mathcal{M}$ may be written in the closed form 


$$
\begin{aligned}
& R(v-w-\mathrm{i} \hbar) T_{-}^{1}(v) \stackrel{2}{\mathcal{M}}(w)=\stackrel{2}{\mathcal{M}}(w) R^{\eta}\left(v-w+\frac{2}{N} \mathrm{i} \hbar\right) T_{-}^{1}(v) \chi(v-w), \\
& R(v-w) T_{+}^{1}(v) \stackrel{2}{\mathcal{M}}(w) \\
& \quad=\stackrel{2}{\mathcal{M}}(w) R^{\eta}\left(v-w+\left(1+\frac{2}{N}\right) \mathrm{i} \hbar\right) T_{+}^{1}(v) \chi(v-w),
\end{aligned}
$$

and indeed imply (5.15).

The factor $\chi(v)$ in (5.6) is finally fixed from the requirement that the quantum determinants from (5.8) commute with everything such that the relations (5.8) are consistent with the algebra multiplication. It is known $[40,41]$ that the $q d e t T_{ \pm}$span the center of $Y_{ \pm}$respectively, thus $\chi(v)$ must ensure that they also commute with $Y_{\mp}$. An essential identity for this calculation is [42]

$$
\begin{aligned}
\operatorname{qdet} T_{ \pm}(w) A_{N} & =A_{N} \frac{1}{T_{ \pm}}(w) \stackrel{2}{T_{ \pm}}(w-\mathrm{i} \hbar) \ldots \stackrel{N}{T_{ \pm}}(w-(N-1) \mathrm{i} \hbar) \\
& =T_{ \pm}^{N}(w-(N-1) \mathrm{i} \hbar) \ldots T_{ \pm}^{2}(w-\mathrm{i} \hbar) T_{ \pm}^{1}(w) A_{N}
\end{aligned}
$$

where $A_{N}$ denotes the antisymmetrizer in the $N$ auxiliary spaces. Modifying the calculation for the Yangian [42] by using our mixed relations (5.6) finally leads to

$$
A_{N} R_{01}^{\prime} \ldots R_{0 N}^{\prime} A_{N} \stackrel{0}{T_{-}}(v) q \operatorname{qdet} T_{+}(w) A_{N}=\operatorname{qdet} T_{+}(w) A_{N} T_{-}^{0}(v) A_{N} R_{01}^{\prime \prime} \ldots R_{0 N}^{\prime \prime} A_{N},
$$

with

$$
\begin{aligned}
& R_{0 j}^{\prime} \equiv R_{0 j}(v-w+(j-2) \mathrm{i} \hbar) \\
& R_{0 j}^{\prime \prime} \equiv R_{0 j}^{\eta}\left(v-w+\left(j+\frac{2}{N}-1\right) \mathrm{i} \hbar\right) \chi(v-w+(j-1) \mathrm{i} \hbar)
\end{aligned}
$$

Now ${ }^{13}$

$$
A_{N} R_{01}^{\prime} \ldots R_{0 N}^{\prime} A_{N}=\frac{v-w-2 \mathrm{i} \hbar}{v-w-\mathrm{i} \hbar} \prod_{j=1}^{N}(v-w+(j-2) \mathrm{i} \hbar) A_{N},
$$

which together with (5.13) implies

$$
A_{N} R_{01}^{\prime \prime} \ldots R_{0 N}^{\prime \prime} A_{N}=\frac{w-v-N \mathrm{i} \hbar}{w-v-(N-1) \mathrm{i} \hbar} \prod_{j=1}^{N} \chi(v-w+(j-1) \mathrm{i} \hbar) A_{N}
$$

Combining these equations yields the functional equation for $\chi(v)$ which is solved by (5.7). Uniqueness of this solution follows from its normalization at $\mathrm{i} \hbar / v \rightarrow 0_{-}$:

$$
\chi(v) \rightarrow 1-\frac{\mathrm{i} \hbar}{v}\left(1+\frac{2}{N}\right), \quad \text { for } \frac{\mathrm{i} \hbar}{v} \rightarrow 0_{-},
$$

which is required in order to obtain the correct classical limit (5.2) from (5.6).

\footnotetext{
${ }^{13}$ We thank A. Molev for pointing this out.
} 
It remains to check that the $*$-operation defined by $(5.10)$ is indeed a conjugate-linear anti-multiplicative automorphism of the structure (5.5)-(5.9). Compatibility of (5.5) and (5.6) with (5.10) obviously follows from $R(\bar{u})=-\overline{R(-u)}, R^{\eta}(\bar{u})=-\overline{R^{\eta}(-u)}$, $\chi(\vec{u})=\overline{\chi(u)}$ and the fact that $R$ and $R^{\eta}$ are symmetric under permutation of the two spaces. Invariance of the restriction of unit quantum determinant (5.8) under $*$ follows from

$$
\begin{aligned}
\operatorname{qdet}\left(T_{ \pm}(w)\right)^{*} & =\sum_{\sigma \in \mathfrak{S}_{N}} \operatorname{sgn}(\sigma) T_{\mp}^{N \sigma(N)}(\bar{w}) \ldots T_{\mp}^{1 \sigma(1)}(\bar{w}+(N-1) \mathrm{i} \hbar) \\
& =\operatorname{qdet}\left(T_{\mp}(\bar{w}+(N-1) \mathrm{i} \hbar)\right)
\end{aligned}
$$

where for the second identity we have employed one of the many properties of the quantum determinant [42]. Finally, compatibility of the symmetry relation (5.9) with $*$ follows directly from invariance of this relation under $*$ :

$$
\left(T_{+}(w) T_{-}^{t}(w)\right)^{*}=\left(T_{+}(w) T_{-}^{t}(w)\right)^{t}=T_{+}(w) T_{-}^{t}(w) \quad \text { for } w \in \mathbb{R} .
$$

The algebra (5.5)-(5.9) is a new structure which in fact brings together some concepts which arose within the last years in the theory of quantum groups. The exchange relations (5.5) define the Yangian $Y(g l(N))[2,3] .{ }^{14}$ The definition of the quantum determinant (5.8) is known from the Yangian $Y(\mathfrak{s l}(N))$ [40-42].

The shift of the arguments of the quantum $R$-matrices in (5.14) reminds the central extension of the normal Yangian double, which has been introduced for quantum affine algebras in [9] and explicitly for the Yangian double in $[44,45]$. In contrast to the usual case, a common shift of $c_{1}$ and $c_{2}$ is no automorphism of (5.5)-(5.10). Rather, the absolute value of both parameters is fixed from the requirement of compatibility with (5.9). From the abstract point of view the central extension takes the critical value at which (5.15) holds, i.e. any representation of the algebra (5.5), (5.6) factorizes over $\mathcal{I}$. The normal (untwisted) Yangian double has a critical value of the central extension at which it possesses an infinite dimensional center [9]. As we shall discuss in the next section, for $N=2$ the algebra $(5.5)-(5.8)$ is in fact isomorphic (but not *-isomorphic) to the normal centrally extended Yangian double at the critical level.

The essential new ingredient of (5.5), (5.6) is the twist ${ }^{15} \eta$ in the mixed relations which already appeared in the classical Poisson algebra. It is basically this peculiarity which requires a new representation theory to be developed.

\footnotetext{
${ }^{14}$ In fact, the underlying algebra in our case is a degeneration $\mathcal{A}_{\hbar}(\mathfrak{s} l(N))$ of the scaling limit of the elliptic affine algebra $\mathcal{A}_{p, q}(s \mathfrak{s}(N))$. In terms of the generating functions $T_{ \pm}(w)$ the exchange relations of this algebra coincide with the Yangian double; however with different analyticity properties. Whereas for the Yangian double the $T_{ \pm}(w)$ are assumed to allow Laurent series in 0 and $\infty$, respectively, they behave analytically in the upper and lower half of the complex plane for $\mathcal{A}_{\hbar}(\mathfrak{s l}(N))$, respectively [43]. This is the case in our model. It suggests to choose Fourier coefficients of $T_{ \pm}(w)$ on the real line as convenient objects for further study of the algebra.

${ }^{15}$ We are aware that the notation of "twist" has been introduced in several contexts for quantum groups in general and even for the Yangians in particular. In Ref. [46], e.g., the "twisted Yangian" denotes the Yangian for the algebra $5 a(N)$. However, we hope that our notation here will not cause undue confusion.
} 
Remark 2. The algebra (5.5)-(5.9) is isomorphic under rescaling of $\hbar$ with positive real numbers. Namely, this is absorbed by a rescaling of the spectral parameter $w$. Negative or complex rescaling in contrast would violate the assumed holomorphy behavior of the classical limit of the $T_{ \pm}(w)$. Depending on the sign of $\hbar$, there is hence a $\mathbb{Z}_{2}$-freedom in constructing (5.5) $-(5.9)$. This corresponds to the symmetry $(+\leftrightarrow-)$ of the classical Poisson algebra (5.1)-(5.4), which is obviously broken after quantization. This freedom might eventually be fixed by the requirement of the existence of unitary representations.

Remark 3. The symmetry property (5.9) together with the definition of the *-map guarantees that the object $\mathcal{M}(w)$ is symmetric and invariant under $*$. In a unitary representation it will thus form a self-adjoint operator. As such it is the natural quantum object that according to (3.7) underlies the original classical field on the symmetry axis. It satisfies the closed exchange relations

$$
\begin{aligned}
& R(v-w) \stackrel{1}{\mathcal{M}}(v) R^{\eta}\left(w-v+\left(1+\frac{2}{N}\right) \mathrm{i} \hbar\right) \stackrel{2}{\mathcal{M}}(w) \\
& \quad=\stackrel{2}{\mathcal{M}}(w) R^{\eta}\left(v-w+\left(1+\frac{2}{N}\right) \mathrm{i} \hbar\right) \stackrel{1}{\mathcal{M}}(v) R(w-v) \frac{\chi(v-w)}{\chi(w-v)},
\end{aligned}
$$

which may be viewed as the quantization of (3.12).

\section{2. $\mathfrak{g}=\mathfrak{s l}(2)$}

To illustrate these formulas we will now discuss the particular case $\mathfrak{g}=\mathfrak{s l}(2)$. This is the model which describes the two Killing vector field reduction of pure $4 \mathrm{~d}$ Einstein gravity and correspondingly already deserves strong interest from the point of view of quantum gravity. The corresponding quantum model has been introduced in [19]. Remarkably in this case there is an algebra isomorphism between our twisted and the normal Yangian double, however this is no $*$-algebra isomorphism.

The exchange relations here read

$$
\begin{gathered}
R(v-w) T_{ \pm}^{1}(v) \stackrel{2}{T_{ \pm}}(w)=T_{ \pm}^{2}(w) \stackrel{1}{T_{ \pm}}(v) R(v-w) \\
R(v-w-\mathrm{i} \hbar) \stackrel{1}{T_{-}}(v) \stackrel{2}{T_{+}}(w)=\stackrel{2}{T_{+}}(w) \stackrel{1}{T_{-}}(v) R^{\eta}(v-w+\mathrm{i} \hbar) \chi(v-w)
\end{gathered}
$$

with $R$ and $R^{\eta}$ from (5.7), where the permutation operator $\Pi$ and its twisted analog $\Pi^{\eta}$ are given by

$$
\Pi \equiv\left(\begin{array}{cccc}
1 & 0 & 0 & 0 \\
0 & 0 & 1 & 0 \\
0 & 1 & 0 & 0 \\
0 & 0 & 0 & 1
\end{array}\right), \quad \Pi^{\eta} \equiv I-\Pi^{\tilde{f}} \equiv\left(\begin{array}{cccc}
0 & 0 & 0 & -1 \\
0 & 1 & 0 & 0 \\
0 & 0 & 1 & 0 \\
-1 & 0 & 0 & 0
\end{array}\right)
$$

Moreover, $\chi$ may be evaluated from (5.7),

$$
\chi(v)=\frac{v(v-2 \mathrm{i} \hbar)}{(v-\mathrm{i} \hbar)(v+\mathrm{i} \hbar)} .
$$


The quantum determinant is given by

$$
\operatorname{qdet} T_{ \pm}(w) \equiv T_{ \pm}^{11}(w-\mathrm{i} \hbar) T_{ \pm}^{22}(w)-T_{ \pm}^{12}(w-\mathrm{i} \hbar) T_{ \pm}^{21}(w)=1 ;
$$

the matrix product

$$
\mathcal{M}(w) \equiv T_{+}(w) T_{-}^{t}(w)=\mathcal{M}(w)^{t}
$$

is symmetric under transposition and satisfies (5.17).

The particular case $N=2$ is distinguished from the higher $N$ already on the classical level by the fact that the involution $\eta$ is an inner automorphism generated by conjugation with

$$
\sigma_{2}=\left(\begin{array}{cc}
0 & \mathrm{i} \\
-\mathrm{i} & 0
\end{array}\right) .
$$

This allows us to "retwist" the mixed relations (5.19) by the following transformation:

$$
\widetilde{T}_{+}(w) \equiv T_{+}(w) \sigma_{2}, \quad \widetilde{T}_{-}(w) \equiv T_{-}(w) .
$$

These retwisted generators satisfy the exchange relations of the normal Yangian double:

$$
\begin{aligned}
& R(v-w) \stackrel{\frac{1}{T_{ \pm}}}{ \pm}(v) \stackrel{2}{\widetilde{T}_{ \pm}}(w)=\widetilde{T}_{ \pm}^{2}(w) \widetilde{T}_{ \pm}(v) R(v-w), \\
& R(v-w-\mathrm{i} \hbar) \stackrel{1}{\tilde{T}_{-}}(v) \tilde{T}_{+}^{2}(w)=\tilde{T}_{+}^{2}(w) \tilde{T}_{-}^{1}(v) R(v-w+\mathrm{i} \hbar) \times(v-w)
\end{aligned}
$$

at the critical level $k=-2$. At this level the center of the Yangian double becomes infinite-dimensional and is generated by the trace of the quantum current [9]

$$
L(w) \equiv\left[\widetilde{T}_{+}(w) \tilde{T}_{-}^{-1}(w)\right] .
$$

Evaluating this in terms of our matrix $\mathcal{M}(w)$ from (5.21) yields

$$
\operatorname{tr} L(w)=\mathcal{M}^{12}(w)-\mathcal{M}^{21}(w) .
$$

The central extension of our structure was precisely determined by the requirement (5.15). Since for $N=2$ the subspace $\mathcal{I}$ is one-dimensional, (5.15) and an explicit calculation shows that it even lies in the center of the algebra (5.18)-(5.19). Here we see complete agreement with the normal Yangian double at the critical level. We have thus equivalence of the twisted structure (5.18), (5.19) with the untwisted (5.23), (5.24), however supplied with a somewhat peculiar *-structure,

$$
\widetilde{T}_{+}(w)^{*}=\widetilde{T}_{-}(\bar{w}) \sigma_{2}
$$

For higher $N$ this equivalence does not hold. Neither is there an algebra isomorphism between (5.5), (5.6) and the normal Yangian double, nor does a center emerge at our critical level, rather criticality is expressed by $(5.15)$. 
Remark 4. For explicit calculations it is sometimes useful to express the exchange relations $(5.18),(5.19)$ in matrix components $T_{ \pm}^{a b}(w)$. The mixed relations (5.19) e.g. may equivalently be written as

$$
\begin{aligned}
(1 & \left.-\frac{(\mathrm{i} \hbar)^{2}}{(v-w)^{2}}\right) T_{-}^{a b}(v) T_{+}^{c d}(w)=\left(1-\frac{\mathrm{i} \hbar}{(v-w)}\right) T_{+}^{c d}(w) T_{-}^{a b}(v) \\
& +\frac{\mathrm{i} \hbar}{(v-w)}\left(T_{+}^{a d}(w) T_{-}^{c b}(v)+\delta^{b d} T_{+}^{c n t}(w) T_{-}^{a m}(v)\right) \\
& +\frac{(\mathrm{i} \hbar)^{2}}{(v-w)^{2}} \delta^{b d}\left(T_{+}^{a m}(w) T_{-}^{c m}(v)-T_{+}^{c m}(w) T_{-}^{a m}(v)\right) .
\end{aligned}
$$

Interpreting the matrix entries of the $T_{ \pm}$as creation and annihilation operators, respectively, the r.h.s. of (5.27) can be viewed as sort of normal ordering.

\section{Outlook}

We have given a complete reformulation of the classical models in terms of the transition matrices of the associated linear system. In contrast to the situation in general integrable models, here the transition matrices themselves are integrals of motion. Moreover they contain a complete set of conserved charges related to the values of the physical fields on the axis $\rho=0$. The Poisson algebra of these matrices has been shown to form a semi-classical version of the Yangian double modified by appearance of a twist by the coset involution $\eta$. The transitive action of the Geroch group becomes manifest and rather transparent in this picture as the Lie-Poisson action generated by the transition matrices. This classical picture has been established for an arbitrary semisimple Lie algebra $\mathfrak{g}$ underlying the $\mathbf{G} / \mathbf{H}$ coset $\sigma$-model.

Quantization for $\mathfrak{g}=\mathfrak{s l}(N)$ led to a twisted Yangian double with central extension where the exact value of the central extension is uniquely determined from consistency, more precisely from compatibility of the structure with the symmetry of the matrix $\mathcal{M}$. For $\mathfrak{g}=\mathfrak{s l}(2)$ the structure is in fact isomorphic (but not $*$-isomorphic) to the centrally extended Yangian double at the critical level with infinite-dimensional center.

Continuation of the program is straightforward to outline. The representation theory of the algebra (5.5)-(5.9) must be studied. So far only the finite-dimensional representations of the normal Yangian are completely understood and classified [47]. These results might serve as basic tools to support the first steps in exploring the relevant infinite-dimensional representations of our algebra. The hope is certainly that the requirement of unitarity with respect to the $*$-structure $(5.10)$ will strongly restrict the choice of representations. In [19] we have suggested a particular Fock space type representation where inspired by the linear truncation of the model the two Yangian halves of (5.5) act as creation and annihilation operators, respectively. However, unitarity of this representation is not obvious, so eventually one might have to face states of negative norm; this remains to be investigated. 
Once the set of possible representations has been identified and hopefully been brought to a minimum the next goal is the construction of coherent states in this framework. These states should exhibit minimal quantum fluctuations around given classical solutions. With them at hand one would finally be in a position to study in detail how quantization affects the known classical solutions of gravity. In particular, this might shed some light on the discussion about the existence and properties of suited coherent states in the truncation of the model to collinearly polarized gravitational waves $[48,49]$. The quantum analog of the Geroch group is supposed to play the key role of a spectrum generating group, i.e. in accordance with the classical picture it should act transitively among the coherent states. It may be possible to shortcut the explicit construction of representation and coherent states by properly understanding the quantization [35] of this Lie-Poisson action that we have described in the classical picture.

Further open problems remain. At this stage we do not know the explicit link of the canonical approach adopted here to the isomonodromic quantization proposed in [50,51] for the same model. Although related quantum group structures appear, the isomonodromic framework is formulated in terms of different observables, which makes the comparison even on the classical level rather non-trivial.

Since the classical picture is already formulated for an arbitrary semisimple Liealgebra $\mathfrak{g}$ there remains the obvious task to elaborate the quantization for higherdimensional coset spaces. As mentioned several times before, this corresponds to the models which descend from dimensional reduction of matter-coupled gravities and supergravities. The study of their quantization so far suffers simply from the fact that the theory of Yangians associated to the exceptional groups, e.g., is still strongly underdeveloped - not to mention their representation theory. To describe the corresponding reduction of maximally extended $N=8$ supergravity [14] one would have to construct the related possibly centrally extended Yangian double of $E_{8(+8)}$ with a twist characterizing the maximal compact subgroup $S O(16)$.

Another highly interesting generalization would be the extension of this framework to a dimensional reduction which includes a timelike Killing vector field. At present it seems rather subtle to rigorously establish a canonical framework in a sector of stationary solutions where the canonical time dependence has been dropped by hand. On the other hand, it is certainly this sector which contains the most interesting physical solutions, in particular the black holes. Justifying the relevance of the fundamental structures obtained in this paper within that context would open the doors to a profound understanding of quantum black holes.

\section{Acknowledgements}

We would like to thank A. Kitaev, H. Nicolai, V. Schomerus and M. Semenov-TianShansky for helpful discussions at different stages of this work. The work of D.K. was partially supported by DFG Contract Ni 290/5-1. H.S. thanks Studienstiftung des deutschen Volkes for support. 


\section{Appendix A. Non-ultralocal term and Poisson structure of transition matrices with and without dilaton-gravity coupled}

Here we will show how the presence of the gravitational and the dilaton field kills all ambiguities in the calculation of the Poisson structure between transition matrices. To make the difference to the two-dimensional sigma models in flat space more manifest, we shall first recall the standard difficulties arising in these models.

\section{A.1. Principal chiral field model (PCM)}

For simplicity we shall consider the principal chiral field model defined by the Lagrangian

$$
\mathcal{L}=\operatorname{tr} J_{\mu} J^{\mu}
$$

with currents $J_{\mu}=G^{-1} \partial_{\mu} G$ for $G \in \mathbf{G}$ and equation of motion

$$
\partial^{\mu} J_{\mu}=0
$$

The original Poisson structure is similar to (2.11),

$$
\begin{aligned}
& \left\{\mathrm{I}_{0}(x), \stackrel{2}{J}_{0}(y)\right\}=\left[\Omega_{\mathfrak{g}}, \stackrel{1}{J}_{0}(x)\right] \delta(x-y), \\
& \left\{\begin{array}{l}
J_{0}(x), \stackrel{2}{J}_{1}(y) \\
J_{0}
\end{array}=\left[\Omega_{\mathfrak{g}}, J_{1}(x)\right] \delta(x-y)-\Omega_{\mathfrak{g}} \partial_{x} \delta(x-y),\right. \\
& \left\{f_{1}(x), \stackrel{2}{J_{1}}(y)\right\}=0 \text {. }
\end{aligned}
$$

For the $L$-operator of the linear system $[26,27]$

$$
L_{1}(x, \lambda) \equiv \frac{2 \lambda}{1-\lambda^{2}}\left(\lambda J_{1}(x)-J_{0}(x)\right)
$$

this implies the following commutation relations:

$$
\begin{aligned}
\left\{L_{1}\left(x, \lambda_{1}\right), \stackrel{2}{L}_{1}\left(y, \lambda_{2}\right)\right\}= & -\frac{4 \lambda_{1} \lambda_{2}}{\left(1-\lambda_{2}^{2}\right)\left(\lambda_{1}-\lambda_{2}\right)}\left[\Omega_{\mathfrak{g}}, \stackrel{1}{L_{1}}\left(\lambda_{1}\right)\right] \delta(x-y) \\
& -\frac{4 \lambda_{1} \lambda_{2}}{\left(1-\lambda_{1}^{2}\right)\left(\lambda_{1}-\lambda_{2}\right)}\left[\Omega_{\mathfrak{g}}, \stackrel{2}{L_{1}}\left(\lambda_{2}\right)\right] \delta(x-y) \\
& -\frac{4 \lambda_{1} \lambda_{2}}{\left(1-\lambda_{1}^{2}\right)\left(1-\lambda_{2}^{2}\right)} \Omega_{\mathfrak{g}} \partial_{x} \delta(x-y)
\end{aligned}
$$

In turn, the transition matrices ${ }^{16}$

\footnotetext{
${ }^{16}$ For clearness we drop the argument $t$ throughout this appendix.
} 


$$
T(x, y, \lambda) \equiv \mathcal{P} \exp \int_{x}^{y} d z L(z, \lambda),
$$

inherit the following Poisson structure [22]:

$$
\begin{aligned}
& \left\{\stackrel{1}{T}\left(x, y, \lambda_{1}\right), \stackrel{2}{T}\left(x^{\prime}, y^{\prime}, \lambda_{2}\right)\right\}=\int_{x}^{y} d z \int_{x^{\prime}}^{y^{\prime}} d z^{\prime}\left(\stackrel{1}{T}(x, z, v) \stackrel{2}{T}\left(x^{\prime}, z^{\prime}, w\right)\right) \\
& \quad \times\left\{L_{1}^{1}\left(z, \lambda_{1}\right), L_{1}^{2}\left(z^{\prime}, \lambda_{2}\right)\right\}\left(\stackrel{1}{T}(z, y, v) \stackrel{2}{T}\left(z^{\prime}, y^{\prime}, w\right)\right),
\end{aligned}
$$

which leads to [23]

$$
\begin{aligned}
& \left\{\stackrel{1}{T}\left(x, y, \lambda_{1}\right), \stackrel{2}{T}\left(x^{\prime}, y^{\prime}, \lambda_{2}\right)\right\} \\
& =\frac{4 \lambda_{1} \lambda_{2}}{\left(\lambda_{1}-\lambda_{2}\right)\left(1-\lambda_{1}^{2}\right)}\left(-\theta\left(x, y^{\prime}, y\right) \stackrel{1}{T}\left(x, y^{\prime}, \lambda_{1}\right) \stackrel{2}{T}\left(x^{\prime}, y^{\prime}, \lambda_{2}\right) \Omega_{\mathfrak{g}} \stackrel{1}{T}\left(y^{\prime}, y, \lambda_{1}\right)\right. \\
& \left.\quad+\theta\left(x, x^{\prime}, y\right) \stackrel{1}{T}\left(x, x^{\prime}, \lambda_{1}\right) \Omega_{\mathfrak{g}} \stackrel{1}{T}\left(x^{\prime}, y, \lambda_{1}\right) \stackrel{2}{T}\left(x^{\prime}, y^{\prime}, \lambda_{2}\right)\right) \\
& \quad+\frac{4 \lambda_{1} \lambda_{2}}{\left(\lambda_{1}-\lambda_{2}\right)\left(1-\lambda_{2}^{2}\right)}\left(-\theta\left(x^{\prime}, y, y^{\prime}\right) \stackrel{1}{T}\left(x, y, \lambda_{1}\right) \stackrel{2}{T}\left(x^{\prime}, y, \lambda_{2}\right) \Omega_{\mathfrak{g}} \stackrel{2}{T}\left(y, y^{\prime}, \lambda_{2}\right)\right. \\
& \quad+\theta\left(x^{\prime}, x, y^{\prime}\right)\left(\stackrel{2}{T}\left(x^{\prime}, x, \lambda_{2}\right) \Omega_{\mathfrak{g}} \stackrel{1}{T}\left(x, y, \lambda_{1}\right) \stackrel{2}{T}\left(x, y^{\prime}, \lambda_{2}\right)\right),
\end{aligned}
$$

where we have made use of the abbreviation:

$$
\theta(x, y, z)= \begin{cases}1 & \text { for } x<y<z \\ 0 & \text { otherwise }(x \neq y \neq z)\end{cases}
$$

The standard source of difficulties is an ambiguity in the limit to coinciding endpoints, which spoils a well-defined calculation of the Poisson algebra of the matrices $T(\lambda) \equiv T(-\infty, \infty, \lambda)$. For example, taking the limit $x, x^{\prime} \rightarrow-\infty, y, y^{\prime} \rightarrow \infty$ assuming that $x<x^{\prime}$ and $y>y^{\prime}$ we get

$$
\left\{\stackrel{1}{T}\left(\lambda_{1}\right), \stackrel{2}{T}\left(\lambda_{2}\right)\right\}=\frac{4 \lambda_{1} \lambda_{2}}{1-\lambda_{1}^{2}}\left[\frac{\Omega_{\mathfrak{g}}}{\lambda_{1}-\lambda_{2}}, \stackrel{1}{T}\left(\lambda_{1}\right) \stackrel{2}{T}\left(\lambda_{2}\right)\right] .
$$

Taking the same limit assuming that $x^{\prime}<x, y<y^{\prime}$ we find a different result:

$$
\left\{\stackrel{1}{T}\left(\lambda_{1}\right), \stackrel{2}{T}\left(\lambda_{2}\right)\right\}=\frac{4 \lambda_{1} \lambda_{2}}{1-\lambda_{2}^{2}}\left[\frac{\Omega_{\mathfrak{g}}}{\lambda_{1}-\lambda_{2}}, \stackrel{1}{T}\left(\lambda_{1}\right) \stackrel{2}{T}\left(\lambda_{2}\right)\right] .
$$

Moreover, both these brackets are obviously not skew-symmetric. Performing the limit with coinciding endpoints is strongly sensitive to the choice of $\theta(x, x, y)$. None of these choices in turn is compatible with Jacobi identities. Several procedures have been suggested to nevertheless give sense to the classical Poisson algebra of the PCM $[17,52,53]$. In the next subsection we shall show that unexpectedly the ambiguity problem turns out to be automatically cured for the dilaton-gravity-coupled model. 


\section{A.2. Algebra of transition matrices in dimensionally reduced gravity}

The calculation of the Poisson algebra of transition matrices is done in a similar way as for the principal chiral model. However, the explicit coordinate dependence of the spectral parameter - related to the presence of gravity and the dilaton field - surprisingly cures the ambiguities we met above. Let $T(x, y, v)$ and $T\left(x^{\prime}, y^{\prime}, w\right)$ be the transition matrices with spectral parameters $v$ and $w$, respectively, and pairwise distinct endpoints $x, y$ and $x^{\prime}, y^{\prime}$. Due to the underlying coset structure of the model, it is a priori not even obvious that the Poisson algebra of the connection $L_{1}$ of the linear system (3.1) is of a closed form. However, this comes out to be true on the constraint surface (2.9):

$$
\begin{aligned}
&\left\{l_{1}\left(z, \gamma_{1}\right), \stackrel{2}{L}_{1}\left(z^{\prime}, \gamma_{2}\right)\right\} \\
&=-\frac{2 \gamma_{1} \gamma_{2}}{\rho\left(\gamma_{1}-\gamma_{2}\right)\left(1-\gamma_{1} \gamma_{2}\right)}\left[\Omega_{\mathfrak{h}}, \stackrel{1}{L}_{1}\left(\gamma_{1}\right)+\stackrel{2}{L_{1}}\left(\gamma_{2}\right)\right] \delta\left(z-z^{\prime}\right) \\
&-\frac{2 \gamma_{2}^{2}\left(1-\gamma_{1}^{2}\right)}{\rho\left(1-\gamma_{2}^{2}\right)\left(\gamma_{1}-\gamma_{2}\right)\left(1-\gamma_{1} \gamma_{2}\right)}\left[\Omega_{\mathfrak{k}}, \stackrel{1}{L}_{1}\left(\gamma_{1}\right)\right] \delta\left(z-z^{\prime}\right) \\
&-\frac{2 \gamma_{1}^{2}\left(1-\gamma_{2}^{2}\right)}{\rho\left(1-\gamma_{1}^{2}\right)\left(\gamma_{1}-\gamma_{2}\right)\left(1-\gamma_{1} \gamma_{2}\right)}\left[\Omega_{\mathfrak{k}}, \stackrel{2}{L_{1}}\left(\gamma_{2}\right)\right] \delta\left(z-z^{\prime}\right) \\
&-\frac{2 \Omega_{\mathfrak{k}}}{\left(1-\gamma_{1}^{2}\right)\left(1-\gamma_{2}^{2}\right)}\left(\frac{\gamma_{1}\left(1+\gamma_{2}^{2}\right)}{\rho(z)}+\frac{\gamma_{2}\left(1+\gamma_{1}^{2}\right)}{\rho\left(z^{\prime}\right)}\right) \partial_{z} \delta\left(z-z^{\prime}\right),
\end{aligned}
$$

with $\gamma_{1} \equiv \gamma(z, v), \gamma_{2} \equiv \gamma\left(z^{\prime}, w\right)$. Inserting this into (A.5) and using (3.2) and (B.6) leads to

$$
\begin{aligned}
& \left\{T(x, y, v), \frac{2}{T}\left(x^{\prime}, y^{\prime}, w\right)\right\}=-\int_{x}^{y} d z \int_{x^{\prime}}^{y^{\prime}} d z^{\prime} \frac{1}{v-w} \delta\left(z-z^{\prime}\right)\left(\partial_{z}+\partial_{z^{\prime}}\right) \Xi_{\mathfrak{h}} \\
& -\int_{x}^{y} d z \int_{x^{\prime}}^{y^{\prime}} d z^{\prime} \frac{2 \gamma_{2}^{2}\left(1-\gamma_{1}^{2}\right)}{\rho\left(1-\gamma_{2}^{2}\right)\left(\gamma_{2}-\gamma_{1}\right)\left(1-\gamma_{1} \gamma_{2}\right)} \delta\left(z-z^{\prime}\right) \partial_{z} \Xi_{\mathfrak{k}} \\
& +\int_{x}^{y} d z \int_{x^{\prime}}^{y^{\prime}} d z^{\prime} \frac{2 \gamma_{1}^{2}\left(1-\gamma_{2}^{2}\right)}{\rho\left(1-\gamma_{1}^{2}\right)\left(\gamma_{1}-\gamma_{2}\right)\left(1-\gamma_{1} \gamma_{2}\right)} \delta\left(z-z^{\prime}\right) \partial_{z^{\prime}} \Xi_{\mathfrak{k}} \\
& -\int_{x}^{y} d z \int_{x^{\prime}}^{y^{\prime}} d z^{\prime} \frac{2\left(\rho^{-1}(z) \gamma_{1}\left(1+\gamma_{2}^{2}\right)+\rho^{-1}\left(z^{\prime}\right) \gamma_{2}\left(1+\gamma_{1}^{2}\right)\right)}{\left(1-\gamma_{1}^{2}\right)\left(1-\gamma_{2}^{2}\right)} \partial_{z} \delta\left(z-z^{\prime}\right) \Xi_{\mathfrak{k}},
\end{aligned}
$$

with

$$
\Xi_{h} \equiv \stackrel{1}{T}(x, z, v) \stackrel{2}{T}\left(x^{\prime}, z^{\prime}, w\right) \Omega_{h} \stackrel{1}{T}(z, y, v) \stackrel{2}{T}\left(z^{\prime}, y^{\prime}, w\right),
$$




$$
\Xi_{\mathfrak{k}} \equiv \stackrel{1}{T}(x, z, v) \stackrel{2}{T}\left(x^{\prime}, z^{\prime}, w\right) \Omega_{\mathfrak{k}} \stackrel{1}{T}(z, y, v) \stackrel{2}{T}\left(z^{\prime}, y^{\prime}, w\right) .
$$

Partial integration of the first three terms reduces the expression to boundary terms, There arise additional terms from derivatives of the spectral parameter (cf. Eq. (B.2)). For example, the second term in (A.9) gives a contribution of

$$
\begin{aligned}
& -\frac{8 \gamma_{1}^{2} \gamma_{2}^{2}\left(\left(\gamma_{1}-\gamma_{2}\right)^{2}+\left(1-\gamma_{1} \gamma_{2}\right)^{2}\right)}{\left(1-\gamma_{1}^{2}\right)\left(1-\gamma_{2}^{2}\right)\left(\gamma_{1}-\gamma_{2}\right)^{2}\left(1-\gamma_{1} \gamma_{2}\right)^{2}} \rho^{-2} \partial_{0} \rho \\
& \quad+\frac{\gamma_{1}\left(1+\gamma_{1}^{2}\right) \gamma_{2}^{2}\left(4 \gamma_{1}\left(\gamma_{1}-\gamma_{2}\right)\left(1-\gamma_{1} \gamma_{2}\right)+2\left(1-\gamma_{1}^{2}\right)\left(1-2 \gamma_{1} \gamma_{2}+\gamma_{2}^{2}\right)\right)}{\left(\gamma_{1}-\gamma_{2}\right)^{2}\left(1-\gamma_{1} \gamma_{2}\right)^{2}\left(1-\gamma_{1}^{2}\right)\left(1-\gamma_{2}^{2}\right)} \rho^{-2} \partial_{1} \rho
\end{aligned}
$$

the third term yields the same with opposite sign and $\gamma_{1}$ and $\gamma_{2}$ interchanged. This combines into a term proportional to $\rho^{-2} \partial_{1} \rho$ which is precisely cancelled by the contribution from the last term in (A.9) (note the different arguments of the dilaton $\rho$ ). Altogether there remain the following boundary terms:

$$
\begin{aligned}
& \left\{\stackrel{1}{T}(x, y, v), \stackrel{2}{T}\left(x^{\prime}, y^{\prime}, w\right)\right\}=\frac{1}{v-w} \\
& \times\left\{\theta\left(x, x^{\prime}, y\right)\left(\stackrel{1}{T}\left(x, x^{\prime}, v\right) \Omega_{\mathfrak{h}} \stackrel{1}{T}\left(x^{\prime}, y, v\right) \stackrel{2}{T}\left(x^{\prime}, y^{\prime}, w\right)\right)\right. \\
& +\theta\left(x^{\prime}, x, y^{\prime}\right)\left(\stackrel{2}{T}\left(x^{\prime}, x, w\right) \Omega_{\mathfrak{h}} \stackrel{1}{T}(x, y, v) \stackrel{2}{T}\left(x, y^{\prime}, w\right)\right) \\
& -\theta\left(x, y^{\prime}, y\right)\left(\stackrel{1}{T}\left(x, y^{\prime}, v\right) \stackrel{2}{T}\left(x^{\prime}, y^{\prime}, w\right) \Omega_{\mathfrak{h}} \stackrel{1}{T}\left(y^{\prime}, y, v\right)\right) \\
& \left.-\theta\left(x^{\prime}, y, y^{\prime}\right)\left(\frac{1}{T}(x, y, v) \stackrel{2}{T}\left(x^{\prime}, y, w\right) \Omega_{\mathfrak{h}} \stackrel{2}{T}\left(y, y^{\prime}, w\right)\right)\right\} \\
& +\frac{\theta\left(x, x^{\prime}, y\right)}{v-w}\left(\stackrel{1}{T}\left(x, x^{\prime}, v\right) \Omega_{\mathfrak{k}} \stackrel{1}{T}\left(x^{\prime}, y, v\right) \stackrel{2}{T}\left(x^{\prime}, y^{\prime}, w\right)\right) \frac{\gamma\left(x^{\prime}, v\right)\left(1-\gamma^{2}\left(x^{\prime}, w\right)\right)}{\gamma\left(x^{\prime}, w\right)\left(1-\gamma^{2}\left(x^{\prime}, v\right)\right)} \\
& +\frac{\theta\left(x^{\prime}, x, y^{\prime}\right)}{v-w}\left(\stackrel{2}{T}\left(x^{\prime}, x, w\right) \Omega_{\mathfrak{k}} \stackrel{1}{T}(x, y, v) \stackrel{2}{T}\left(x, y^{\prime}, w\right)\right) \frac{\gamma(x, w)\left(1-\gamma^{2}(x, v)\right)}{\gamma(x, v)\left(1-\gamma^{2}(x, w)\right)} \\
& -\frac{\theta\left(x, y^{\prime}, y\right)}{v-w}\left(\stackrel{1}{T}\left(x, y^{\prime}, v\right) \stackrel{2}{T}\left(x^{\prime}, y^{\prime}, w\right) \Omega_{\mathfrak{k}} \frac{1}{T}\left(y^{\prime}, y, v\right)\right) \frac{\gamma\left(y^{\prime}, v\right)\left(1-\gamma^{2}\left(y^{\prime}, w\right)\right)}{\gamma\left(y^{\prime}, w\right)\left(1-\gamma^{2}\left(y^{\prime}, v\right)\right)} \\
& -\frac{\theta\left(x^{\prime}, y, y^{\prime}\right)}{v-w}\left(\stackrel{1}{T}(x, y, v) \stackrel{2}{T}\left(x^{\prime}, y, w\right) \Omega_{\mathfrak{k}} \stackrel{2}{T}\left(y, y^{\prime}, w\right)\right) \frac{\gamma(y, w)\left(1-\gamma^{2}(y, v)\right)}{\gamma(y, v)\left(1-\gamma^{2}(y, w)\right)},
\end{aligned}
$$

where the functions $\theta(x, y, z)$ are the same as in (A.6).

The objects of interest now are the modified transition matrices

$$
\mathcal{V}(x) T(x, y, v) \mathcal{V}^{-1}(y) \text {. }
$$

As has been discussed in Section 3, they give rise to constants of motion when $x$ and $y$ approach the spatial boundary. Moreover, these quantities are by definition invariant under the gauge transformations (2.3). Their Poisson bracket gets additional contributions from 


$$
\begin{gathered}
\left\{\stackrel{1}{T}(x, y, v), \stackrel{2}{\mathcal{V}}\left(x^{\prime}\right)\right\}=\frac{2 \theta\left(x, x^{\prime}, y\right) \gamma\left(x^{\prime}, v\right)}{\rho\left(x^{\prime}\right)\left(1-\gamma^{2}\left(x^{\prime}, v\right)\right)} \stackrel{1}{T}\left(x, x^{\prime}, v\right) \stackrel{2}{\mathcal{V}}\left(x^{\prime}\right) \Omega_{\mathfrak{k}} \stackrel{1}{T}\left(x^{\prime}, y, v\right), \\
\left\{\mathcal{V}(x), \stackrel{2}{T}\left(x^{\prime}, y^{\prime}, w\right)\right\}=-\frac{2 \theta\left(x^{\prime}, x, y^{\prime}\right) \gamma(x, w)}{\rho(x)\left(1-\gamma^{2}(x, w)\right)} \stackrel{2}{T}\left(x^{\prime}, x, w\right) \stackrel{1}{\mathcal{V}}(x) \Omega_{\mathfrak{k}} \stackrel{2}{T}\left(x, y^{\prime}, w\right) .
\end{gathered}
$$

etc.

The final result then takes a form which superficially resembles the PCM:

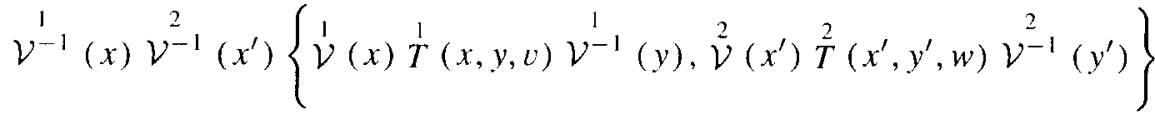

$$
\begin{aligned}
& \times \stackrel{1}{\mathcal{V}}(y) \stackrel{2}{\mathcal{V}}\left(y^{\prime}\right) \\
& =\frac{1}{v-w}\left\{\theta\left(x, x^{\prime}, y\right)\left(\stackrel{1}{T}\left(x, x^{\prime}, v\right) \Omega_{\mathfrak{h}} T^{\prime}\left(x^{\prime}, y, v\right) \stackrel{2}{T}\left(x^{\prime}, y^{\prime}, w\right)\right)\right. \\
& +\theta\left(x^{\prime}, x, y^{\prime}\right)\left(\stackrel{2}{T}\left(x^{\prime}, x, w\right) \Omega_{\mathfrak{h}} \stackrel{1}{T}(x, y, v) \stackrel{2}{T}\left(x, y^{\prime}, w\right)\right) \\
& -\theta\left(x, y^{\prime}, y\right)\left(\stackrel{1}{T}\left(x, y^{\prime}, v\right) \stackrel{2}{T}\left(x^{\prime}, y^{\prime}, w\right) \Omega_{\mathfrak{h}} \stackrel{1}{T}\left(y^{\prime}, y, v\right)\right) \\
& \left.-\theta\left(x^{\prime}, y, y^{\prime}\right)\left(\stackrel{1}{T}(x, y, v) \stackrel{2}{T}\left(x^{\prime}, y, w\right) \Omega_{h} \stackrel{2}{T}\left(y, y^{\prime}, w\right)\right)\right\} \\
& +\frac{\theta\left(x, x^{\prime}, y\right)}{v-w}\left(\stackrel{1}{T}\left(x, x^{\prime}, v\right) \Omega_{\mathrm{k}} \stackrel{1}{T}\left(x^{\prime}, y, v\right) \stackrel{2}{T}\left(x^{\prime}, y^{\prime}, w\right)\right) \\
& \times \frac{1-2 \gamma\left(x^{\prime}, v\right) \gamma\left(x^{\prime}, w\right)+\gamma^{2}\left(x^{\prime}, v\right)}{1-\gamma^{2}\left(x^{\prime}, v\right)} \\
& +\frac{\theta\left(x^{\prime}, x, y^{\prime}\right)}{v-w}\left(\stackrel{2}{T}\left(x^{\prime}, x, w\right) \Omega_{\mathrm{k}} \stackrel{1}{T}(x, y, v) \stackrel{2}{T}\left(x, y^{\prime}, w\right)\right) \\
& \times \frac{1-2 \gamma(x, w) \gamma(x, v)+\gamma^{2}(x, w)}{1-\gamma^{2}(x, w)} \\
& -\frac{\theta\left(x, y^{\prime}, y\right)}{v-w}\left(\stackrel{1}{T}\left(x, y^{\prime}, v\right) \stackrel{2}{T}\left(x^{\prime}, y^{\prime}, w\right) \Omega_{\mathrm{k}} \stackrel{1}{T}\left(y^{\prime}, y, v\right)\right) \\
& \times \frac{1-2 \gamma\left(y^{\prime}, v\right) \gamma\left(y^{\prime}, w\right)+\gamma^{2}\left(y^{\prime}, v\right)}{1-\gamma^{2}\left(y^{\prime}, v\right)} \\
& -\frac{\theta\left(x^{\prime}, y, y^{\prime}\right)}{v-w}\left(\stackrel{1}{T}(x, y, v) \stackrel{2}{T}\left(x^{\prime}, y, w\right) \Omega_{\mathfrak{k}} \stackrel{2}{T}^{2}\left(y, y^{\prime}, w\right)\right) \\
& \times \frac{1-2 \gamma(y, w) \gamma(y, v)+\gamma^{2}(y, w)}{1-\gamma^{2}(y, w)} .
\end{aligned}
$$

In fact, neglecting the coset structure (i.e. formally putting $\Omega_{\mathfrak{h}}=\Omega_{\mathfrak{k}}=\Omega_{\mathfrak{g}}$ ) and replacing the coordinate dependent spectral parameters $\gamma$ by constant ones $\lambda$ (making use of (B.6)), Eq. (A.11) explicitly reduces to (A.6). At first sight, we thus face the same fatal problem shown in (A.7), (A.8) when trying to treat coinciding endpoints.

In the limit to spatial boundaries however, the coordinate dependence of the spectral parameter changes the situation drastically. Let us show this for a spacelike dilaton 
identified with the spatial (radial) coordinate $(\rho=x \in[0, \infty$, cf. Section 3.1). Recall that here we have variables $T_{ \pm}(w)$ where the index \pm denotes the sign of the imaginary part of $w$. According to (3.4) we evaluate (A.11) in the limit $x, x^{\prime} \rightarrow 0, y, y^{\prime} \rightarrow \infty$. The first four terms become

$$
\left[\frac{\Omega_{\mathfrak{h}}}{v-w}, \frac{1}{T}(v) \stackrel{2}{T}(w)\right],
$$

for arbitrary indices \pm at the $T$ 's.

The next two terms show the ambiguous behavior at coinciding endpoints. Just like in (A.7), (A.8) their coefficients tend to

$$
\frac{1}{v-w} \frac{1-2 \gamma\left(x^{\prime}, v\right) \gamma\left(x^{\prime}, w\right)+\gamma^{2}\left(x^{\prime}, v\right)}{1-\gamma^{2}\left(x^{\prime}, v\right)}
$$

and

$$
\frac{1}{v-w} \frac{1-2 \gamma(x, w) \gamma(x, v)+\gamma^{2}(x, w)}{1-\gamma^{2}(x, w)}
$$

respectively, giving different results for different ways of taking the limit $x^{\prime} \rightarrow x$. But now the difference with the PCM becomes manifest: Since the spectral parameters depend on the spatial coordinates, in the limit $x, x^{\prime} \rightarrow 0$ both expressions (A.12) and (A.13) commonly tend to $(v-w)^{-1}$ (cf. (B.5)). Their sum thus is independent of how this limit is taken, keeping e.g. $x<x^{\prime}$ or $x>x^{\prime}$ or also $x=x^{\prime}$ with $\theta(x, x, y) \equiv \frac{1}{2}$.

In a similar way the ambiguity from the last two terms vanishes. In the limit $y, y^{\prime} \rightarrow \infty$ the combinations

$$
\frac{1}{v-w} \frac{1-2 \gamma\left(y^{\prime}, v\right) \gamma\left(y^{\prime}, w\right)+\gamma^{2}\left(y^{\prime}, v\right)}{1-\gamma^{2}\left(y^{\prime}, v\right)}
$$

and

$$
\frac{1}{v-w} \frac{1-2 \gamma(y, w) \gamma(y, v)+\gamma^{2}(y, w)}{1-\gamma^{2}(y, w)}
$$

approach the same value. This common value is sensitive to the choice of indices \pm at the T's. If $\gamma(v)$ and $\gamma(w)$ lie in the same of the two regions $D_{+}$and $D_{-}$they tend to $(v-w)^{-1}$, whereas they tend to $(w-v)^{-1}$ otherwise (cf. (B.5)).

Thus, finally we arrive at the following Poisson structure:

$$
\begin{aligned}
& \left\{\frac{1}{T_{ \pm}}(v), T_{ \pm}^{2}(w)\right\}=\left[\frac{\Omega_{\mathfrak{g}}}{v-w}, T_{ \pm}^{1}(v) \stackrel{2}{T_{ \pm}}(w)\right], \\
& \left\{T_{ \pm}^{1}(v), T_{\mp}^{2}(w)\right\}=\frac{\Omega_{\mathfrak{g}}}{v-w} T_{ \pm}^{1}(v) T_{\mp}^{2}(w)-T_{ \pm}^{1}(v) T_{\mp}^{2}(w) \frac{\Omega_{\mathfrak{g}}^{\eta}}{v-w},
\end{aligned}
$$

with $\Omega_{\mathfrak{g}}^{\eta} \equiv \Omega_{\mathfrak{h}}-\Omega_{\mathfrak{k}}$ obtained from $\Omega_{\mathfrak{g}}$ by applying $\eta$ in one of the two spaces.

Remark 5. The presence of the dilaton field in the original equations of motion (2.13) has given rise to the coordinate dependence of the spectral parameters. We have shown 
how the resulting algebra of transition matrices differs essentially from the related one in the PCM since it does not suffer from any ambiguities. This fact may be summarized as follows: the coordinate dependence of the spectral parameter is inherited by the ambiguities which hence die out at spatial infinity.

Remark 6. This result does not provide a proper regularization of the PCM; switching off dilaton-gravity does not allow a well-defined limit from the equations of motion (2.13) to (A.2). We have seen that the regularity of the algebra of transition matrices heavily relied on the behavior of the dilaton field $\rho$ at the spatial boundaries (cf. (A.12), (A.13) and (B.5)). With constant dilaton field all the ambiguities of the PCM come back. In fact, in the context of dimensionally reduced gravity there are no non-trivial solutions of this model with constant dilaton field due to additional constraints from the gravitational energy-momentum tensor (see, e.g., Ref. [21]).

\section{Appendix B. The spectral parameters}

The variable spectral parameter $\gamma$ is a function of the constant spectral parameter $w$ according to

$$
\gamma(x, t, w)=\gamma\left(\frac{w+\tilde{\rho}}{\rho}\right)=\frac{1}{\rho}\left(w+\tilde{\rho}-\sqrt{(w+\tilde{\rho})^{2}-\rho^{2}}\right) .
$$

It satisfies the differential equations

$$
\gamma^{-1} \partial_{\mu} \gamma=\frac{1+\gamma^{2}}{1-\gamma^{2}} \rho^{-1} \partial_{\mu} \rho+\frac{2 \gamma}{1-\gamma^{2}} \epsilon_{\mu \nu} \rho^{-1} \partial^{\nu} \rho,
$$

as well as

$$
\gamma^{-1} \partial_{w} \gamma=-\frac{2 \gamma}{\rho\left(1-\gamma^{2}\right)}
$$

The inverse formula reads

$$
w=\frac{1}{2} \rho\left(\gamma+\frac{1}{\gamma}\right)-\tilde{\rho}
$$

The parameter $\gamma$ lives on the Riemann surface defined by $\sqrt{(w+\tilde{\rho}+\rho)(w+\tilde{\rho}-\rho)}$, which is a twofold covering of the complex $w$-plane with $x^{\mu}$-dependent branch cut. Transition between the two sheets corresponds to $\gamma \rightarrow \frac{1}{\gamma}$. The branch cut connects the points $w=-\tilde{\rho} \pm \rho$ on the real $w$-axis, which correspond to $\gamma(w=-\tilde{\rho} \pm \rho)= \pm 1$. The real $w$ with $|w+\tilde{\rho}|<|\rho|$ are mapped onto the unit circle $|\gamma|=1$. Real $w$ with $|w+\tilde{\rho}|>|\rho|$ are mapped onto the real $\gamma$-axis. Dividing the $w$-plane into two regions $H_{ \pm}$and the $\gamma$-plane into four regions $D_{ \pm}, \tilde{D}_{ \pm}$according to Fig. B.1, $D_{ \pm}$and $\tilde{D}_{ \pm}$lie over $H_{ \pm}$, respectively. 

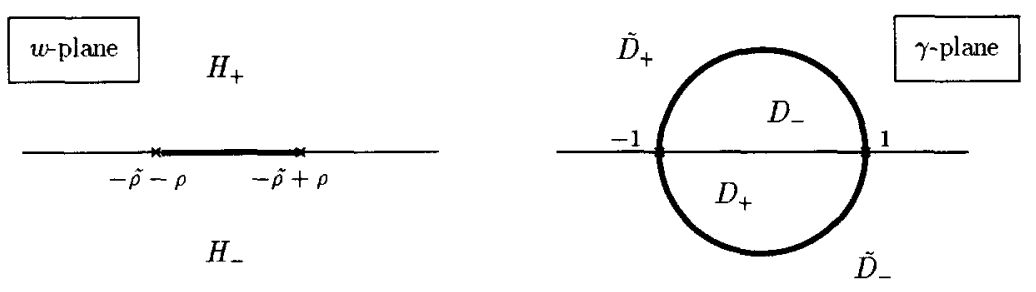

Fig. B.1. The spectral parameter planes,

In particular, it is important that for fixed $w \notin \mathbb{R}$ and continuously varying $\rho$ and $\tilde{\rho}$ the parameter $\gamma$ moves within a fixed of these regions. The limits of these trajectories are given by

$$
\gamma(\rho \rightarrow 0) \rightarrow\left\{\begin{array}{c}
0 \\
\infty
\end{array}, \quad \gamma(\rho \rightarrow \infty) \rightarrow\left\{\begin{array}{c}
\mathrm{i} \\
-\mathrm{i}
\end{array}, \quad \gamma(\tilde{\rho} \rightarrow \pm \infty) \rightarrow\left\{\begin{array}{c}
0 \\
\infty
\end{array}\right.\right.\right.
$$

Another useful formula for two spectral parameters $\gamma(x, t, v)$ and $\gamma(x, t, w)$ at coinciding coordinates $x, t$ is

$$
v-w=\frac{\rho}{2} \frac{(\gamma(v)-\gamma(w))(\gamma(v) \gamma(w)-1)}{\gamma(v) \gamma(w)}
$$

\section{References}

[1] E. Sklyanin, On the complete integrability of the Landau-Lifshitz equation, preprint LOMI E-3-79 (1979).

|2| L. Faddeev, E. Sklyanin and L. Takhtajan, Quantum inverse scattering method, Theor. Math. Phys. 40 (1979) 194.

13] V. Drinfeld, Hopf algebras and the quantum Yang-Baxter equation, Sov. Math. Dokl. 32 (1985) 254.

[4] D. Bernard, Hidden Yangians in 2d massive current algebras, Commun. Math. Phys. 137 (1991) 191.

[5] P. Bouwknegt and K. Schoutens, The $S U(n)-1$ WZW models: Spinon decomposition and Yangian structure, Nucl. Phys. B 482 (1996) 345.

16] V. Drinfeld, Quantum groups, in Proc. Int. Congress Math., Berkeley 1986 (AMS, 1986) p. 798.

17| A. LeClair and F.A. Smirnov, Infinite quantum group symmetry of fields in massive $2 d$ quantum field theory, Int. J. Mod. Phys. A 7 (1992) 2997.

181 D. Bernard and A. Leclair, The quantum double in integrable quantum field theory, Nucl. Phys. B 399 (1993) 709 .

$19 \mid$ N. Reshetikhin and M. Semenov-Tian-Shansky, Central extensions of quantum current groups, Lett. Math. Phys. 19 (1990) 133.

[10] K. Kuchař, Canonical quantization of cylindrical gravitational waves, Phys. Rev. D 4 (1971) 955.

[11] A. Ashtekar and M. Pierri, Probing quantum gravity through exactly soluble midisuperspaces 1, J. Math. Phys. 37 (1996) 6250.

|12| W. Kinnersley and D.M. Chitre, Symmetries of the stationary Einstein-Maxwell field equations II, J. Math. Phys. 18 (1977) 1538.

[13] D. Gal'tsov and O. Kechkin, Hidden symmetries in dilaton-axion gravity, in Geometry and Integrable Models, ed. P. Pyatov and S. Solodukhin, (World Scientific, Singapore, 1996).

$114 \mid$ B. Julia, Application of supergravity to gravitation theories, in Unified field theories in more than 4 dimensions, ed. V.D. Sabbata and E. Schmutzer (World Scientific, Singapore, 1983) p. 215.

| 15] F. Ernst, New formulation of the axially symmetric gravitational field problem, Phys. Rev. 167 (1968) 1175 . 
[16] M. Lüscher and K. Pohlmeyer, Scattering of massless lumps and non-local charges in the two-dimensional classical non-linear $\sigma$-model, Nucl. Phys. B 137 (1978) 46.

1 17 | L. Faddeev and N. Reshetikhin. Integrability of the principal chiral field model in $1+1$ dimension, Ann. Phys. 167 (1985) 227.

1 18| D. Korotkin and H. Samtleben. Poisson realization and quantization of the Geroch group, Class. Quant. Grav. 14 ( 1997) L151.

$119 \mid$ D. Korotkin and H. Samtleben. Canonical quantization of cylindrical gravitational waves with two polarizations, Phys. Rev. Lett. 80 (1998) 14.

[20] P. Breitenlohner and D. Maison, On the Geroch group, Ann. Inst. H. Poincaré. Phys. Théor. 46 (1987) 215.

121] H. Nicolai, Two-dimensional gravities and supergravities as integrable systems, in Recent Aspects of Quantum Fields, ed. H. Mitter and H. Gausterer (Springer, Berlin, 1991).

122/ L. Faddeev and L. Takhtajan, Hamiltonian Methods in the Theory of Solitons (Springer, Berlin, 1987).

123] $\mathbf{H}$. de Vega, H. Eichenherr and $\mathbf{J}$. Maillet, Classical and quantum algebras of non-local charges in $\sigma$ models, Commun. Math. Phys. 92 (1984) 507.

$124 \mathrm{I} \mathrm{V}$. Belinskii and V. Zakharov, Integration of the Einstein equations by means of the inverse scattering problem technique and construction of exact soliton solutions, Sov. Phys. JETP 48 (1978) 985.

$125 \mid$ D. Maison, Are the stationary, axially symmetric Einstein equations completely integrable?, Phys. Rev. Lett. 41 (1978) 521.

|26| K. Pohlmeyer, Integrable Hamiltonian systems and interactions through quadratic constraints, Commun. Math. Phys. 46 (1976) 207.

|27| V. Zakharov and A. Mikhailov, Relativistically invariant two-dimensional models of field theory which are integrable by means of the inverse scattering problem method, Sov. Phys. JETP 47 (1978) 1017.

|28| R. Gowdy, Vacuum space-times with two-parameter spacelike isometry group and compact invariant hypersurfaces: topologies and boundary conditions, Ann. Phys. 83 (1974) 203.

$[29 \mid$ V. Husain, Einstein's equations and the chiral model, Phys. Rev. D 53 (1996) 4327.

[30] G.A. Mena Marugán, Canonical quantization of the Gowdy model, Phys. Rev. D 56 (1997) 908.

131| M. Cavaglià. V. de Alfaro and A.T. Filippov, Hamiltonian formalism for black holes and quantization II, Int. J. Mod. Phys. D 5 ( 1996) 227.

|32| R. Geroch, A method for generating new solutions of Einstein's equations II, J. Math. Phys. 13 (1972) 394.

[33] M. Semenov-Tian-Shansky, Dressing transformations and Poisson group actions, Publ. RIMS, Kyoto Univ. 21 (1985) 1237.

| 34 | O. Babelon and D. Bernard, Dressing symmetries, Commun. Math. Phys. 149 (1992) 279.

[35] J.-H. Lu, Moment maps at the quantum level, Commun. Math. Phys. 157 (1993) 389.

136| I. Hauser and F. Ernst, A homogeneous Hilbert problem for the Kinnersley-Chitre transformations, J. Math. Phys. 21 (1980) 1126.

137| Y.-S. Wu and M.-L. Ge, A simplified derivation of the Geroch group in two-dimensional reduced gravity, J. Math. Phys. 24 (1983) 1187.

[38] L.-L. Chau and M.-L. Ge, Kac-Moody algebra from infinitesimal Riemann-Hilbert transform, J. Math. Phys. 30 (1988) 166.

139| B. Julia and H. Nicolai, Conformal internal symmetry of $2 \mathrm{~d}$ sigma models coupled to gravity and a dilaton. Nucl. Phys. B 482 (1996) 431.

140| A. Izergin and V. Korepin, A lattice model related to the non-linear Schrödinger equation, Sov. Math. Doc. 26 (1981) 653.

$141]$ P. Kulish and E. Sklyanin, Quantum spectral transform method. Recent developments, in Integrable Quantum Field Theories, ed. J. Hietarinta and C. Montonen, Vol. 151, Lecture Notes in Physics (Springer, Berlin, 1982) p. 61.

142] A. Molev, M. Nazarov and G. Olshanskii, Yangians and classical Lie algebras, Russ. Math. Surveys 51 (1996) 205 .

|43| S. Khoroshkin, D. Lebedev and S. Pakuliak, Yangian algebras and classical Riemann problems, preprint ITEP-TH-66/97, q-alg/97/2057 ( 1997).

$144 \mid$ S. Khoroshkin. Central extension of the Yangian double, in Collection SMF, Colloque Septièmes Rencontres du Contact Franco-Belge en Algèbre, Reins, preprint q-alg/9602031 ( I996).

|4.5| K. Iohara and M. Kohno. A central extension of $\mathcal{D} Y_{\hbar}\left(\mathfrak{g l}_{2}\right)$ and its vertex representations, Lett. Math. Phys. 37 ( 1996) 319. 
[46] G. Olshanskii, Twisted Yangians and infinite-dimensional classical Lie algebras, in Quantum Groups, ed. P. Kulish, No. 1510, Lecture Notes in Math. (Springer, Berlin, 1992) p. 103.

[47] V. Chari and A. Pressley, A Guide to Quantum Groups (Cambridge Univ. Press, Cambridge, 1994).

[48| A. Ashtekar, Large quantum gravity effects: Unforeseen limitations of the classical theory, Phys. Rev. Lett. 77 (1996) 4864.

[49| R. Gambini and J. Pullin, Large quantum gravity effects: Back reaction on matter, Mod. Phys. Lett. A 12 ( 1997$) 2407$.

$150 \mid$ D. Korotkin and H. Nicolai, Isomonodromic quantization of dimensionally reduced gravity, Nucl. Phys. B 475 (1996) 397.

151] D. Korotkin and H. Samtleben, Quantization of coset space sigma models coupled to two-dimensional gravity, Commun. Math. Phys. 190 (1997) 411.

152| A. Duncan, H. Nicolai and M. Niedermaier, On the Poisson bracket algebra of monodromy matrices, $Z$. Phys. C 46 (1990) 147.

1531 N. MacKay, On the classical origins of Yangian symmetry in integrable field theory, Phys. Lett. B 28I (1992) 90 . 